Henrique S. Brito ${ }^{1}$, Eliana V. Carraça ${ }^{2}$, António L. Palmeira ${ }^{2}$, José P. Ferreira ${ }^{3}$, Veronica Vleck ${ }^{1}$ Duarte Araújo $^{1}$

1. CIPER, Faculdade de Motricidade Humana, Universidade de Lisboa, Cruz Quebrada - Dafundo, 1499-002, Lisbon, Portugal 2. CIDEFES, Faculdade de Educação Física e Desporto, Universidade Lusófona de Humanidades e Tecnologias, Campo Grande, 1749-024, Lisbon, Portugal

3. CIDAF, Faculdade de Ciências do Desporto e Educação Física, Universidade de Coimbra, Estádio Universitário de Coimbra, 3040-248, Coimbra, Portugal

\title{
Benefits to Performance and Well-being of Nature-based Exercise: A Critical Systematic Review and Meta-analysis
}

1 table - Table S1 - Design, samples, interventions, settings, outcomes, and results of the included studies 


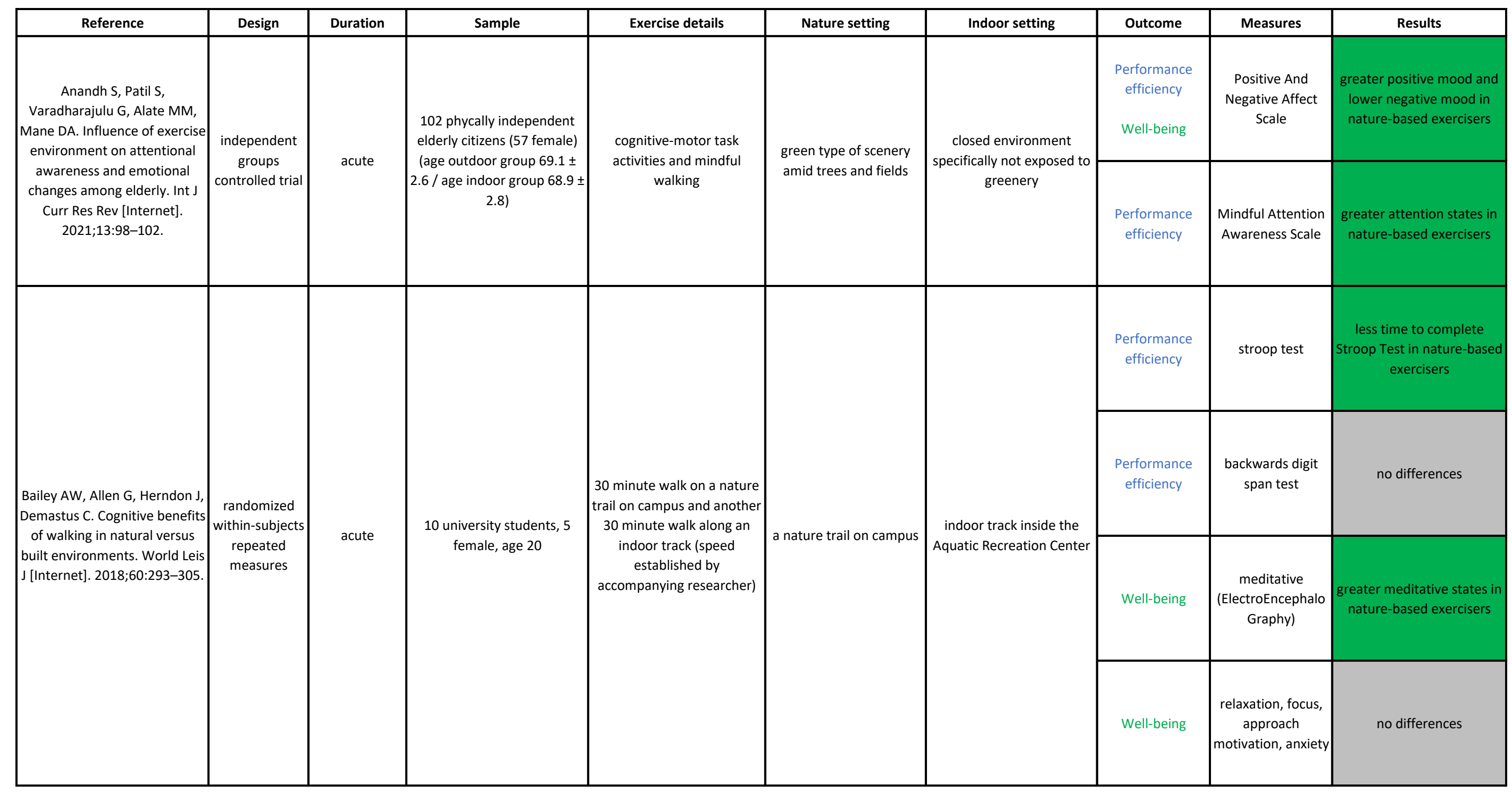




\begin{tabular}{|c|c|c|c|c|c|c|c|c|c|}
\hline \multirow{3}{*}{$\begin{array}{c}\text { Byrka K, Ryczko N. Positive } \\
\text { effects of dancing in natural } \\
\text { versus indoor settings: The } \\
\text { mediating role of engagement } \\
\text { in physical activity. J Environ } \\
\text { Psychol [Internet]. Elsevier Ltd; } \\
\text { 2018;57:25-33. }\end{array}$} & \multirow{3}{*}{$\begin{array}{l}\text { randomized } \\
\text { independent } \\
\text { groups }\end{array}$} & \multirow{3}{*}{ acute } & \multirow{3}{*}{$\begin{array}{c}64 \text { dancers with atleast } 3 \\
\text { months of salsa experience, } \\
51 \text { female, age } 29.4 \pm 8.9\end{array}$} & \multirow{3}{*}{$\begin{array}{l}40 \text { minute dancing session } \\
\text { in a park and in a dance } \\
\text { room; self-selected intensity }\end{array}$} & \multirow{3}{*}{\begin{tabular}{|} 
square of the Southern \\
Park in Wroctaw on \\
hardgravel ground, \\
surrounded by a knee- \\
high wall and oldtrees on \\
two sides.
\end{tabular}} & \multirow{3}{*}{$\begin{array}{c}\text { rooms were approximately } \\
30 \text { square meters in size, } \\
\text { with 4-meter-high ceilings, } \\
\text { mirrors on one wall and } \\
\text { windows on the other }\end{array}$} & $\begin{array}{l}\text { Performance } \\
\text { efficacy }\end{array}$ & Accelerometer & $\begin{array}{l}\text { greater engagement in } \\
\text { nature-based exercisers }\end{array}$ \\
\hline & & & & & & & Well-being & \begin{tabular}{|l|} 
positive emotions \\
(Exercise-Induced \\
Feeling Inventory)
\end{tabular} & no differences \\
\hline & & & & & & & $\begin{array}{l}\text { Performance } \\
\text { efficiency }\end{array}$ & \begin{tabular}{|c} 
exhaustion \\
(Exercise-Induced \\
Feeling Inventory)
\end{tabular} & $\begin{array}{l}\text { lower exhaustion in nature- } \\
\text { based exercisers }\end{array}$ \\
\hline \multirow{4}{*}{$\begin{array}{c}\text { Calogiuri G, Evensen K, } \\
\text { Weydahl A, Andersson K, Patil } \\
\text { G, Ihlebæk C, et al. Green } \\
\text { exercise as a workplace } \\
\text { intervention to reduce job } \\
\text { stress. Results from a pilot } \\
\text { study. Work. 2016; }\end{array}$} & \multirow{4}{*}{$\begin{array}{l}\text { randomized } \\
\text { independent } \\
\text { groups }\end{array}$} & \multirow{4}{*}{ acute } & \multirow{4}{*}{$\begin{array}{l}14 \text { healthy office employees, } \\
\text { sedentary or moderately } \\
\text { active, } 7 \text { females, age } 49 \pm 8\end{array}$} & \multirow{4}{*}{$\begin{array}{l}\text { a biking session (25-minutes } \\
\text { - } 6082 \mathrm{~m} \text { ), and strength } \\
\text { session (eight exercises } \\
\text { covering all the major } \\
\text { muscular groups) using } \\
\text { elastic rubber bands with } \\
\text { handles (20-minutes). self- } \\
\text { perceived moderate } \\
\text { intensity during the warm- } \\
\text { up and the cool-down parts } \\
\text { and a moderately high } \\
\text { intensity during the work- } \\
\text { out }\end{array}$} & \multirow{4}{*}{$\begin{array}{c}\text { The nature group biked } \\
\text { along a track in a forest } \\
\text { area nearby both } \\
\text { workplaces. The } \\
\text { rubberbands session took } \\
\text { place in a grass-yard of } \\
\text { one of the workplaces } \\
\text { just outside the forest } \\
\text { area }\end{array}$} & \multirow{4}{*}{$\begin{array}{c}\text { The indoor setting aimed } \\
\text { to reproduce a 'typical' } \\
\text { exercise setting (gym-hall). }\end{array}$} & $\begin{array}{l}\text { Performance } \\
\text { efficiency }\end{array}$ & $\begin{array}{c}\text { Heart Rate - } \\
\text { estimated marginal } \\
\text { mean }(\%)\end{array}$ & no differences \\
\hline & & & & & & & Well-being & $\begin{array}{c}\text { Fascination, Being } \\
\text { Away (Perceived } \\
\text { Restorativeness } \\
\text { Scale) }\end{array}$ & $\begin{array}{l}\text { greater fascination and } \\
\text { being away in nature-based } \\
\text { exercisers }\end{array}$ \\
\hline & & & & & & & Well-being & $\begin{array}{c}\text { positive affect } \\
\text { (Physical Activity } \\
\text { Affect Scale) }\end{array}$ & $\begin{array}{l}\text { marginally greater positive } \\
\text { affect in nature-based } \\
\text { exercisers }\end{array}$ \\
\hline & & & & & & & Well-being & $\begin{array}{c}\text { tranquility } \\
\text { (Physical Activity } \\
\text { Affect Scale) }\end{array}$ & no differences \\
\hline
\end{tabular}




\begin{tabular}{|c|c|c|c|c|c|c|c|c|c|}
\hline $\begin{array}{l}\text { Chu CY, Lu SY, Lin KF. } \\
\text { Influences of Exercise } \\
\text { Experience and Exercise } \\
\text { Settings on Heart Rate } \\
\text { Responses During Self-selected } \\
\text { Intensity Exercises. J Exerc Sci } \\
\text { Fit. 2010;8:73-7. }\end{array}$ & $\begin{array}{c}\text { counterbalance } \\
\mathrm{d} \text { crossover } \\
\text { controlled trial }\end{array}$ & acute & $\begin{array}{c}26 \text { healthy male adults, age } \\
22.3 \pm 0.7 \text { years }\end{array}$ & $\begin{array}{l}20 \text { minutes of continuous } \\
\text { running at a self-selected } \\
\text { exercise intensity }\end{array}$ & $\begin{array}{l}\text { outdoor track field } \\
\text { setting }\end{array}$ & indoor treadmill setting & $\begin{array}{l}\text { Performance } \\
\text { efficiency }\end{array}$ & heart rate & $\begin{array}{c}\text { lower Heart rate in nature- } \\
\text { based exercisers }\end{array}$ \\
\hline \multirow{3}{*}{$\begin{array}{l}\text { Crossan C, Salmoni A. A } \\
\text { Simulated Walk in Nature: } \\
\text { Testing Predictions From the } \\
\text { Attention Restoration Theory. } \\
\text { Environ Behav [Internet]. } \\
\text { 2021;53:277-95. }\end{array}$} & \multirow{3}{*}{$\begin{array}{c}\text { randomized } \\
\text { counterbalance } \\
d \text { crossover }\end{array}$} & \multirow{3}{*}{$\begin{array}{l}\text { acute (nature } \\
\text { simulation) }\end{array}$} & \multirow{3}{*}{$\begin{array}{l}22 \text { university participants (13 } \\
\text { female) age } 23 \text { years old }\end{array}$} & \multirow{3}{*}{$\begin{array}{l}10 \text { minute treadmill walk at } \\
\text { self-selected speed viewing } \\
\text { nature VR or blank screen }\end{array}$} & \multirow{3}{*}{$\begin{array}{l}\text { large, 180-degree screen } \\
\text { with the projection of an } \\
\text { unfolding, simulated } \\
\text { nature walk through a } \\
\text { forest }\end{array}$} & \multirow{3}{*}{$\begin{array}{l}\text { large, 180-degree blank } \\
\text { white screen }\end{array}$} & $\begin{array}{l}\text { Performance } \\
\text { efficiency }\end{array}$ & $\begin{array}{l}\text { Backwards Digit } \\
\text { Span Test }\end{array}$ & $\begin{array}{l}\text { greater working memory } \\
\text { Performance in nature- } \\
\text { based exercisers }\end{array}$ \\
\hline & & & & & & & $\begin{array}{l}\text { Performance } \\
\text { efficiency }\end{array}$ & necker cube test & no differences \\
\hline & & & & & & & Well-being & $\begin{array}{l}\text { Positive and } \\
\text { Negative Affect } \\
\text { Scale }\end{array}$ & no differences \\
\hline \multirow{2}{*}{\begin{tabular}{|} 
Duncan MJ, Clarke ND, Birch \\
SL, Tallis J, Hankey J, Bryant E, \\
et al. The effect of green \\
exercise on blood pressure, \\
heart rate and mood state in \\
primary school children. Int J \\
Environ Res Public Health \\
[Internet]. 2014;11:3678-88.
\end{tabular}} & \multirow{2}{*}{$\begin{array}{c}\text { counterbalance } \\
\mathrm{d} \text { crossover } \\
\text { controlled trial }\end{array}$} & \multirow{2}{*}{$\begin{array}{l}\text { acute (nature } \\
\text { simulation) }\end{array}$} & \multirow{2}{*}{$\begin{array}{c}14 \text { children, } 7 \text { female, age } 10 \\
\pm 1 \text { years old }\end{array}$} & \multirow{2}{*}{$\begin{array}{c}\text { cycled for } 15 \mathrm{~min} \text { at a } \\
\text { moderate intensity } \\
\text { (50\%HRR) }\end{array}$} & \multirow{2}{*}{$\begin{array}{c}\text { watching a film of cycling } \\
\text { in a forest environment }\end{array}$} & \multirow{2}{*}{ viewing a blank screen } & $\begin{array}{l}\text { Performance } \\
\text { efficiency }\end{array}$ & Heart Rate (bpm) & no differences \\
\hline & & & & & & & Well-being & $\begin{array}{l}\text { Brunel Mood State } \\
\text { Inventory }\end{array}$ & no differences \\
\hline
\end{tabular}




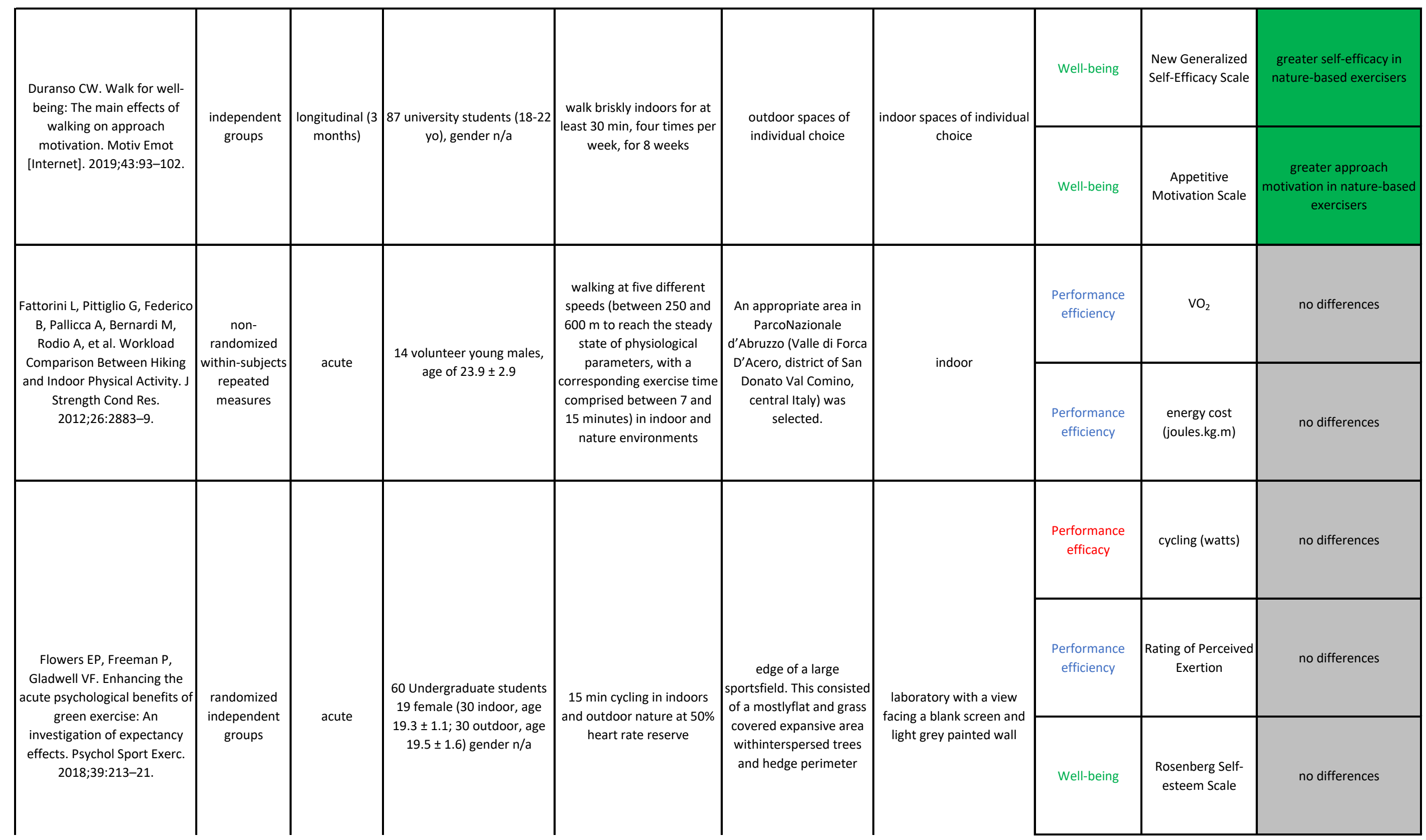




\begin{tabular}{|c|c|c|c|c|c|c|c|c|c|}
\hline & & & & & & & $\begin{array}{l}\text { Performance } \\
\text { efficiency }\end{array}$ & $\begin{array}{l}\text { vigour (Profile of } \\
\text { Mood States) }\end{array}$ & $\begin{array}{l}\text { greater vigor in nature- } \\
\text { based exercise }\end{array}$ \\
\hline \multirow{5}{*}{$\begin{array}{l}\text { Focht BC. Brief walks in } \\
\text { outdoor and laboratory } \\
\text { environments: Effects on } \\
\text { affective responses, } \\
\text { enjoyment, and intentions to } \\
\text { walk for exercise. Res Q Exerc } \\
\text { Sport. United States; } \\
\text { 2009;80:611-20. }\end{array}$} & \multirow{5}{*}{$\begin{array}{l}\text { randomized } \\
\text { counter- } \\
\text { balanced } \\
\text { repeated } \\
\text { measures }\end{array}$} & \multirow{5}{*}{ acute } & \multirow{5}{*}{$\begin{array}{l}35 \text { physically active college- } \\
\text { age women (age } 22.1 \pm 1.7 \text { ) }\end{array}$} & \multirow{5}{*}{$\begin{array}{l}\text { 10-min walks (self-selected } \\
\text { intensity) }\end{array}$} & \multirow{5}{*}{$\begin{array}{l}\text { standardized route on } \\
\text { sidewalks and walking } \\
\text { paths in the area } \\
\text { immediately surrounding } \\
\text { the building }\end{array}$} & \multirow{5}{*}{ laboratory } & $\begin{array}{l}\text { Performance } \\
\text { efficiency }\end{array}$ & $\% \operatorname{HRmax}$ & no differences \\
\hline & & & & & & & Well-being & Feeling Scale & $\begin{array}{l}\text { greater affect in nature- } \\
\text { based exercise }\end{array}$ \\
\hline & & & & & & & $\begin{array}{l}\text { Performance } \\
\text { efficiency }\end{array}$ & Felt Arousal Scale & $\begin{array}{l}\text { greater arousal in nature- } \\
\text { based exercise }\end{array}$ \\
\hline & & & & & & & Well-being & $\begin{array}{l}\text { revitalization, } \\
\text { positive } \\
\text { engagement } \\
\text { (Exercise-Induced } \\
\text { Feeling Inventory) }\end{array}$ & $\begin{array}{l}\text { greater revitalization and } \\
\text { positive engagement in } \\
\text { nature-based exercise }\end{array}$ \\
\hline & & & & & & & $\begin{array}{l}\text { Performance } \\
\text { efficiency }\end{array}$ & $\begin{array}{l}\text { exhaustion } \\
\text { (Exercise-Induced } \\
\text { Feeling Inventory) }\end{array}$ & no differences \\
\hline
\end{tabular}




\begin{tabular}{|c|c|c|c|c|c|c|c|c|c|}
\hline \multirow{3}{*}{$\begin{array}{c}\text { Harte JL, Eifert GH. The effects } \\
\text { of running, environment, and } \\
\text { attentional focus on athletes' } \\
\text { catecholamine and cortisol } \\
\text { levels and mood. } \\
\text { Psychophysiology. United } \\
\text { States; 1995;32:49-54. }\end{array}$} & \multirow{3}{*}{$\begin{array}{l}\text { non- } \\
\text { randomized } \\
\text { counter- } \\
\text { balanced } \\
\text { repeated } \\
\text { measures }\end{array}$} & \multirow{3}{*}{ acute } & \multirow{3}{*}{$\begin{array}{c}10 \text { male amateur triathletes } \\
\text { or marathon runners (age } \\
27,1 \text { ) }\end{array}$} & \multirow{3}{*}{$\begin{array}{c}12-\mathrm{km} \text { run outdoors (about } \\
45 \text { minutes to complete) or } \\
45 \mathrm{~min} \text { run on treadmill } \\
\text { (warm-up period, running } \\
\text { for } 15 \mathrm{~min} \text { at } 10-12 \mathrm{~km} / \mathrm{hr}, \\
\text { and then a } 30-\mathrm{min} \\
\text { run at } 15-18 \mathrm{~km} / \mathrm{hr} \text { - just for } \\
\text { treadmill run) }\end{array}$} & \multirow{3}{*}{$\begin{array}{c}\text { designated route around } \\
\text { James Cook University } \\
\text { campus }\end{array}$} & \multirow{3}{*}{$\begin{array}{c}\text { 6- } x \text { 6-m sports physiology } \\
\text { laboratory with brick walls } \\
\text { and high-set windows }\end{array}$} & Well-being & $\begin{array}{c}\text { tension, } \\
\text { depression, anger, } \\
\text { fatigue (Profile of } \\
\text { Mood States) }\end{array}$ & $\begin{array}{l}\text { lower tension, depression, } \\
\text { anger, fatigue, and greater } \\
\text { vigor in nature-based } \\
\text { exercisers }\end{array}$ \\
\hline & & & & & & & Well-being & $\begin{array}{c}\text { confusion / } \\
\text { bewilderment } \\
\text { (Profile of Mood } \\
\text { States) }\end{array}$ & no differences \\
\hline & & & & & & & $\begin{array}{l}\text { Performance } \\
\text { efficiency }\end{array}$ & $\begin{array}{c}\text { Rating of Perceived } \\
\text { Exertion }\end{array}$ & $\begin{array}{c}\text { lower perceived exertion in } \\
\text { nature-based exercisers }\end{array}$ \\
\hline $\begin{array}{c}\text { Hopker JG, Coleman DA, Wiles } \\
\text { JD, Galbraith A. Familiarisation } \\
\text { and reliability of sprint test }\end{array}$ & randomized & acute & 38 team sport players, age & $\begin{array}{c}\text { four indoor sprint trials } \\
\text { (treadmill) and four outdoor }\end{array}$ & outdoor field hard court & Indor laboratory & $\begin{array}{l}\text { Performance } \\
\text { efficacy }\end{array}$ & $\begin{array}{l}\text { seconds to finish } \\
\text { course }\end{array}$ & $\begin{array}{l}\text { Faster sprint time in nature- } \\
\text { based exercisers }\end{array}$ \\
\hline $\begin{array}{l}\text { field assessment. J Sport Sci } \\
\text { Med. 2009;8:528-32. }\end{array}$ & controlled trial & & & of 20 -metres & & & $\begin{array}{l}\text { Performance } \\
\text { efficacy }\end{array}$ & $\begin{array}{c}\text { running peak force } \\
\text { /average force }\end{array}$ & no differences \\
\hline $\begin{array}{c}\text { Irandoust K, Taheri M. The } \\
\text { effect of Vitamin D } \\
\text { supplement and indoor Vs } \\
\text { outdoor physical activity on } \\
\text { depression of obese depressed } \\
\text { women. Asian J Sports Med } \\
\text { [Internet]. 2017;8. }\end{array}$ & $\begin{array}{c}\text { randomised } \\
\text { independent } \\
\text { groups } \\
\text { controlled trial }\end{array}$ & $\begin{array}{l}\text { longitudinal (3 } \\
\text { months) }\end{array}$ & $\begin{array}{c}75 \text { severely depressed obese } \\
\text { women with Vitamin D } \\
\text { deficiency, age } 43.2 \pm 12.4 \\
\text { years }\end{array}$ & $\begin{array}{c}50 \text { minutes of main protocol } \\
\text { (treadmill versus outdoor } \\
\text { jogging) four times a week } \\
\text { for } 3 \text { months at target heart } \\
\text { rate of } 55 \%-75 \% \text { (11 to } 12 \\
\text { on the RPE scale) }\end{array}$ & several outdoor spaces & several indoor spaces & Well-being & $\begin{array}{l}\text { Beck depression } \\
\text { inventory }\end{array}$ & no differences \\
\hline
\end{tabular}




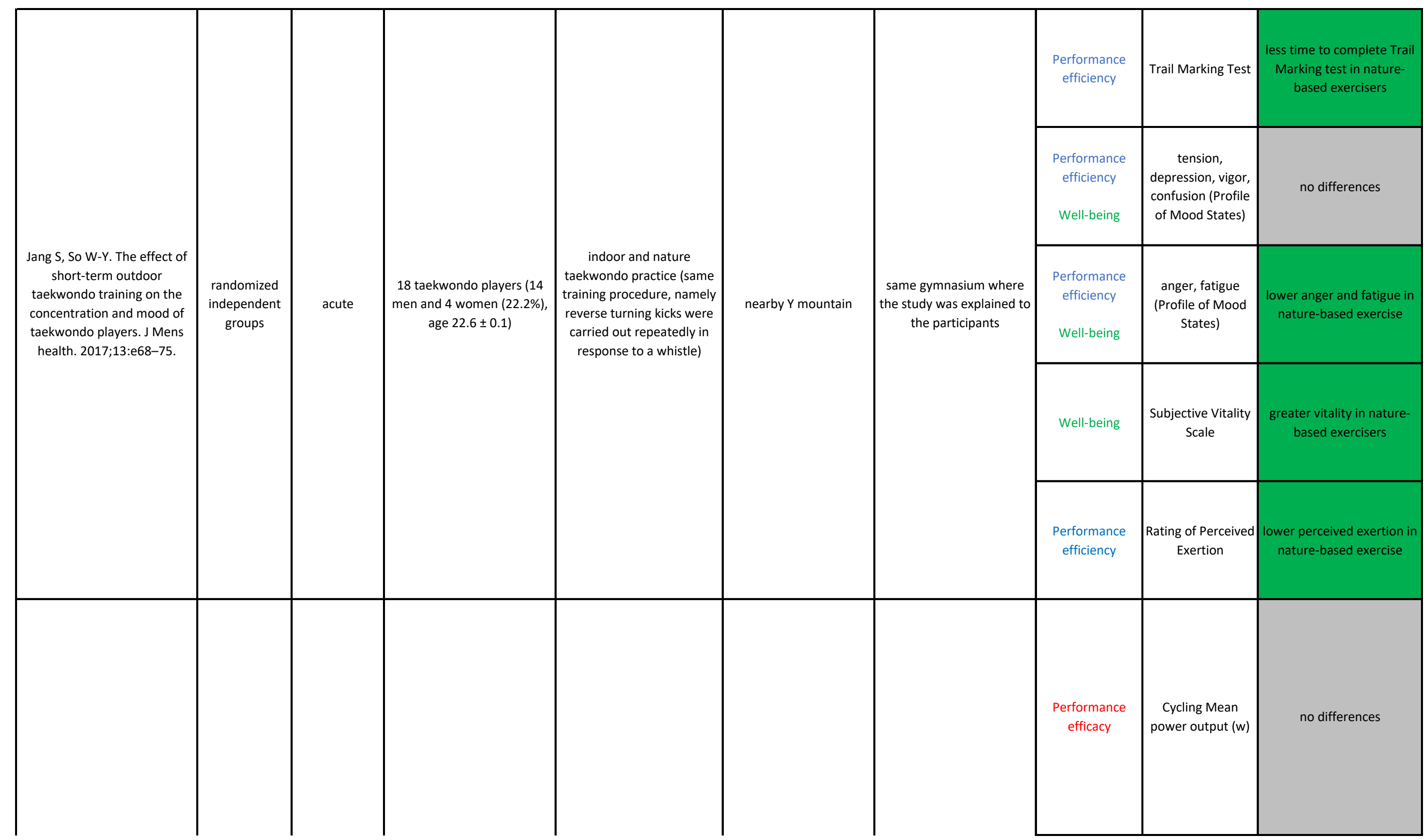


Jeffries, $O$, Waldron, $M$ Patterson, S. D., and Galna, B. (2019). An Analysis of

Variability in Power Output randomized

During Indoor and Outdoo within-sub repeated

20 male cyclists, age $36 \pm 9$ 20-min time-trial cycling (cadence was freely chosendependant on their preferred pacing strategy)

Sports Physiol. Perform. 14, 1273-1279 cycle-specific, traffic-free race circuit. The track measured $1.52 \mathrm{~km}$ in distance, $6 \mathrm{~m}$ wide, with $\sim 4 \mathrm{~m}$ total elevation gain per lap and 7 shallow corners that allowed continuous pe

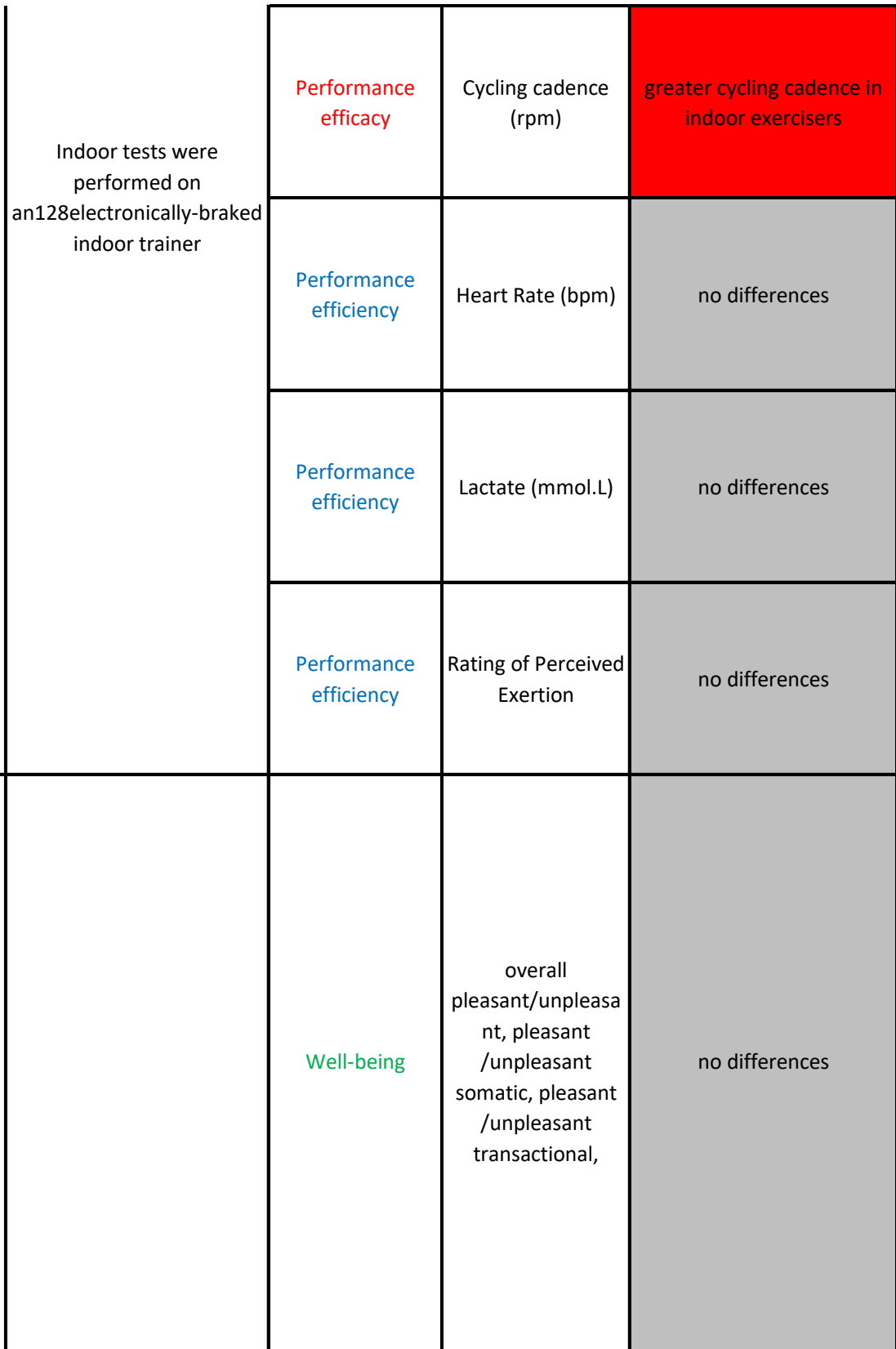




\begin{tabular}{|c|c|c|c|c|c|c|c|c|c|}
\hline \multirow[t]{4}{*}{$\begin{array}{l}\text { Kerr JH, Fujiyama H, Sugano A, } \\
\text { Okamura T, Chang M, Onouha } \\
\text { F. Psychological responses to } \\
\text { exercising in laboratory and } \\
\text { natural environments. Psychol } \\
\text { Sport Exerc. 2006;7:345-59. }\end{array}$} & \multirow[t]{4}{*}{$\begin{array}{l}\text { non- } \\
\text { randomized } \\
\text { counter- } \\
\text { balanced } \\
\text { repeated } \\
\text { measures }\end{array}$} & \multirow[t]{4}{*}{ acute } & \multirow[t]{4}{*}{$\begin{array}{l}22 \text { male students } \\
\text { (recreational runners), age } \\
22.7 \pm 1.7 \text { ) and } 22 \text { male } \\
\text { students (competitive } \\
\text { runners), age } 20.6 \pm 1.3 \text { ) }\end{array}$} & \multirow[t]{4}{*}{$\begin{array}{c}5 \mathrm{~km} \text { running indoors on a } \\
\text { treadmill in a laboratory and } \\
\text { outdoors in a natural } \\
\text { environment. A moderate } \\
\text { running intensity, } \\
\text { representing } 60 \% \text { heart rate } \\
\text { reserve }\end{array}$} & \multirow[t]{4}{*}{$\begin{array}{c}\text { a tree-lined footpath } \\
\text { beside a road which } \\
\text { circled the university } \\
\text { campus. The outdoor } \\
\text { running pathway ran } \\
\text { alongside two small } \\
\text { lakes, through woods and } \\
\text { playing fields and the } \\
\text { road had only occasional } \\
\text { traffic }\end{array}$} & \multirow[t]{4}{*}{$\begin{array}{c}\text { university sports medicine } \\
\text { laboratory }\end{array}$} & Well-being & $\begin{array}{c}\text { relaxation, anxiety, } \\
\text { excitement, } \\
\text { boredom, placidity, } \\
\text { anger, } \\
\text { provocativeness, } \\
\text { sullenness, } \\
\text { humiliation, } \\
\text { modesty, shame... }\end{array}$ & no differences \\
\hline & & & & & & & Well-being & pride & $\begin{array}{l}\text { higher levels of pride for } \\
\text { recreational nature-based } \\
\text { exercisers }\end{array}$ \\
\hline & & & & & & & Well-being & $\begin{array}{c}\text { tension external, } \\
\text { somatic; effort } \\
\text { external, somatic }\end{array}$ & no differences \\
\hline & & & & & & & Well-being & $\begin{array}{c}\text { tension and effort } \\
\text { external }\end{array}$ & $\begin{array}{l}\text { lower tension and effort } \\
\text { stress in competitive indoor } \\
\text { exercisers; no differences in } \\
\text { recreative exercisers }\end{array}$ \\
\hline $\begin{array}{l}\text { Kim T, Lee S, Assoc Comp M. } \\
\text { Restorative Effects of Exercise } \\
\text { in Virtual Environments } \\
\text { [Internet]. New York: Assoc } \\
\text { Computing Machinery; } 2018 .\end{array}$ & $\begin{array}{l}\text { non- } \\
\text { randomized } \\
\text { counter- } \\
\text { balanced } \\
\text { repeated } \\
\text { measures }\end{array}$ & $\begin{array}{l}\text { acute (nature } \\
\text { simulation) }\end{array}$ & $\begin{array}{c}24 \text { undergraduate students } \\
\text { for participants, } 9 \text { female, } \\
\text { age } n / a\end{array}$ & $\begin{array}{c}150 \text { second (participants } \\
\text { should ride for } 22-25 \mathrm{~km} / \mathrm{h} \text { ) } \\
\text { cycling ride indoors and } \\
\text { virtual reality (nature) }\end{array}$ & $\begin{array}{c}\text { Virtual nature (watched } \\
\text { virtual environments } \\
\text { video using HMD) }\end{array}$ & indoor & Well-being & $\begin{array}{c}\text { calmness } \\
\text { (Activation- } \\
\text { Deactivation } \\
\text { Adjective Checklist) }\end{array}$ & $\begin{array}{l}\text { greater calmness in nature- } \\
\text { based exercisers }\end{array}$ \\
\hline
\end{tabular}




\begin{tabular}{|c|c|c|c|c|c|c|c|c|c|}
\hline \multirow{3}{*}{$\begin{array}{l}\text { LaCaille RA, Masters KS, Heath } \\
\text { EM. Effects of cognitive } \\
\text { strategy and exercise setting } \\
\text { on running performance, } \\
\text { perceived exertion, affect, and } \\
\text { satisfaction. Psychol Sport } \\
\text { Exerc. 2004;5:461-76. }\end{array}$} & \multirow{3}{*}{$\begin{array}{l}\text { randomized } \\
\text { within-subjects } \\
\text { repeated } \\
\text { measures }\end{array}$} & \multirow{3}{*}{ acute } & \multirow{3}{*}{$\begin{array}{c}60 \text { individuals, } 38 \text { female, } \\
\text { who ran an average distance } \\
\text { of at least } 15 \text { miles per week, } \\
\text { age } 26.8 \text { years } \pm 8.9\end{array}$} & \multirow{3}{*}{$\begin{array}{l}\text { running a distance of } 5 \mathrm{~km} \\
\text { indoors and outdoors (run } \\
\text { as fast as they would like) }\end{array}$} & \multirow{3}{*}{$\begin{array}{c}\text { a } 5 \mathrm{~km} \text { outdoor flat road } \\
\text { route }\end{array}$} & \multirow{3}{*}{$\begin{array}{l}\text { indoor } 200 \mathrm{~m} \text { track and a } \\
\text { standard motor-driven } \\
\text { treadmill in a university } \\
\text { exercise laboratory }\end{array}$} & $\begin{array}{l}\text { Performance } \\
\text { efficacy }\end{array}$ & $\begin{array}{l}\text { time to complete } \\
\text { course (minutes) }\end{array}$ & $\begin{array}{c}\text { less time to complete } \\
\text { distance by nature-based } \\
\text { exercisers }\end{array}$ \\
\hline & & & & & & & $\begin{array}{l}\text { Performance } \\
\text { efficiency }\end{array}$ & $\left|\begin{array}{c}\text { Rating of Perceived } \\
\text { Exertion }\end{array}\right|$ & $\begin{array}{l}\text { lower perceived exertion in } \\
\text { nature-based exercise }\end{array}$ \\
\hline & & & & & & & $\begin{array}{l}\text { Performance } \\
\text { efficiency } \\
\text { Well-being }\end{array}$ & $\begin{array}{l}\text { Exercise-Induced } \\
\text { Feeling Inventory }\end{array}$ & $\begin{array}{l}\text { greater positive affect in } \\
\text { nature-based exercisers }\end{array}$ \\
\hline \multirow{3}{*}{$\begin{array}{l}\text { Lacharité-lemieux M, Dionne } \\
\text { IJ. Physiological Responses to } \\
\text { Indoor Versus Outdoor } \\
\text { Training in Postmenopausal } \\
\text { Women. J Aging Phys Act. } \\
\text { 2016;24:275-83. }\end{array}$} & \multirow{3}{*}{$\begin{array}{l}\text { randomized } \\
\text { independent } \\
\text { groups }\end{array}$} & \multirow{3}{*}{$\begin{array}{l}\text { longitudinal (3 } \\
\text { months) }\end{array}$} & \multirow{3}{*}{$\begin{array}{c}23 \text { healthy to overweight } \\
\text { post-menopausal women } \\
\text { (age } 60.7 \pm 4.8 \text { ) }\end{array}$} & \multirow{3}{*}{$\begin{array}{l}\text { three weekly 1-hr sessions } \\
\text { of mixed training (aerobic } \\
\text { and resistance) conducted } \\
\text { outdoors (OutTr) or indoors } \\
\text { (InTr), but was otherwise } \\
\text { identical; self-selected } \\
\text { intensity but within } 65 \% \text { - } \\
95 \% \text { of maximal heart rate } \\
\text { during the training duration }\end{array}$} & \multirow{3}{*}{$\begin{array}{c}\text { esthetically pleasing and } \\
\text { mainly natural park } \\
\text { beside a body of water, } \\
\text { where paths were lined } \\
\text { with large trees and rich } \\
\text { biodiversity }\end{array}$} & \multirow{3}{*}{$\begin{array}{l}\text { large meeting room in the } \\
\text { Research Center }\end{array}$} & $\begin{array}{l}\text { Performance } \\
\text { efficiency }\end{array}$ & $\begin{array}{c}\text { Heart Rate (bpm, } \\
\% \text { HRmax) }\end{array}$ & no differences \\
\hline & & & & & & & $\begin{array}{l}\text { Performance } \\
\text { efficacy }\end{array}$ & $\begin{array}{l}\text { bench press (n } \\
\text { reps) }\end{array}$ & $\begin{array}{c}\text { greater endurance (bench } \\
\text { press) for nature-based } \\
\text { exercisers }\end{array}$ \\
\hline & & & & & & & $\begin{array}{l}\text { Performance } \\
\text { efficacy }\end{array}$ & $\begin{array}{l}\text { Leg Press and Lat } \\
\text { pull down (n reps) }\end{array}$ & no differences \\
\hline & & & & & & & Well-being & Feeling Scale & no differences \\
\hline
\end{tabular}




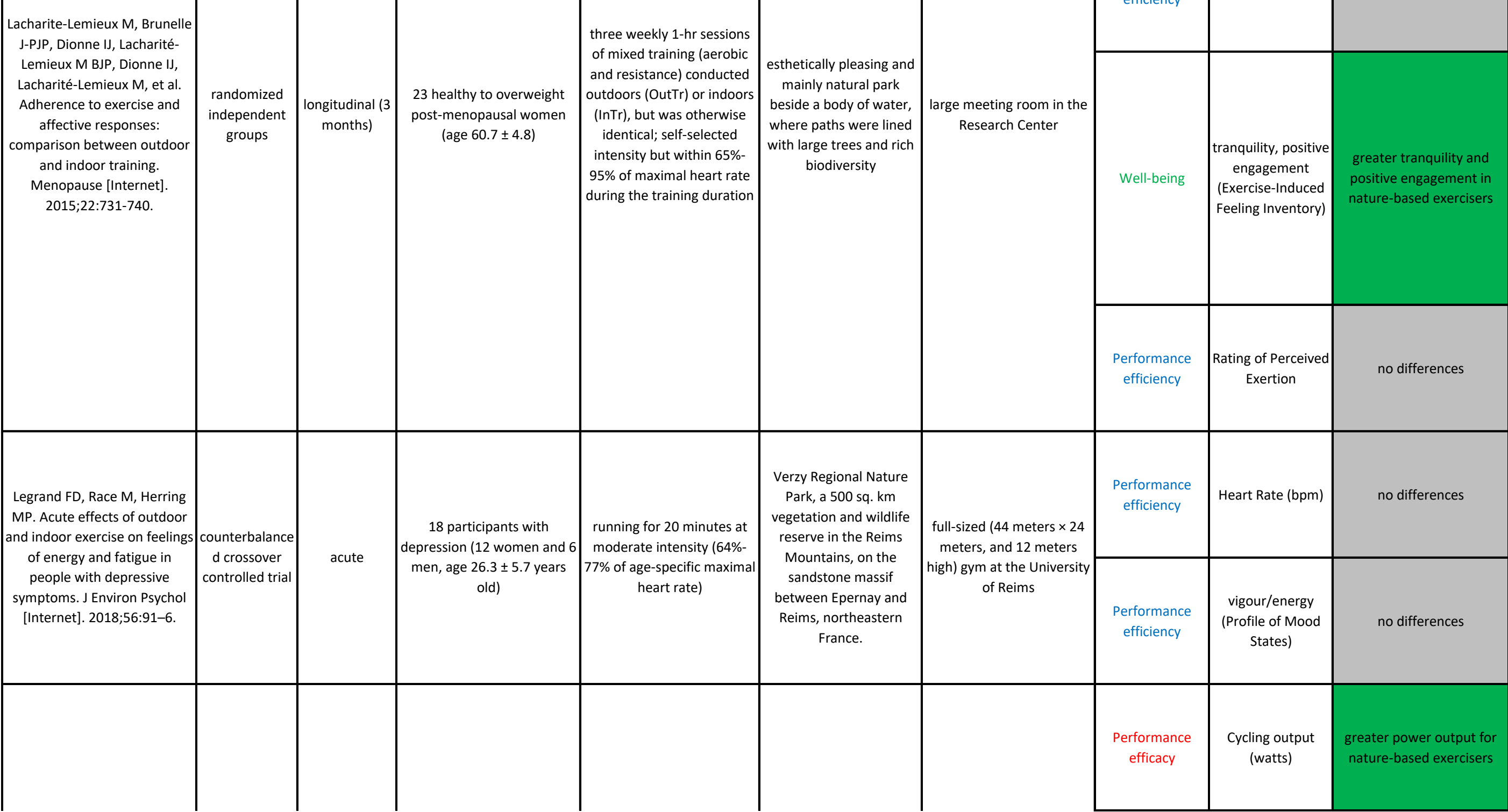




\begin{tabular}{|c|c|c|c|c|c|c|c|c|c|}
\hline \multirow{4}{*}{$\begin{array}{l}\text { Mieras ME, Heesch MWS, } \\
\text { Slivka DR. Physiological and } \\
\text { Psychological Responses to } \\
\text { Outdoor vs. Laboratory } \\
\text { Cycling. J Strength Cond Res. } \\
\text { 2014;28:2324-9. }\end{array}$} & \multirow{4}{*}{$\begin{array}{l}\text { randomized } \\
\text { counter- } \\
\text { balanced } \\
\text { repeated } \\
\text { measures }\end{array}$} & \multirow{4}{*}{ acute } & \multirow{4}{*}{$\begin{array}{l}12 \text { recreationally trained } \\
\text { male cyclists (age } 37 \pm 2 \text { ) }\end{array}$} & \multirow{4}{*}{$\begin{array}{c}\text { cycling at a self-selected } \\
\text { intensity. Participants } \\
\text { completed the trial on their } \\
\text { own bicycle mounted on the } \\
\text { electronically braked cycle } \\
\text { trainer ergometer. The } \\
\text { outdoor trail was recorded } \\
\text { through globalpositioning } \\
\text { system (GPS). The outdoor } \\
\text { trials were completed along } \\
\text { a relatively flat,out, and } \\
\text { back course on a paved } \\
\text { recreation trail }\end{array}$} & \multirow{4}{*}{$\begin{array}{c}\text { relatively flat, out, and } \\
\text { back course on a paved } \\
\text { recreation trail (Keystone } \\
\text { Trail, Omaha, NE, USA) }\end{array}$} & \multirow{4}{*}{$\begin{array}{c}\text { Exercise Physiology } \\
\text { Laboratory }\end{array}$} & $\begin{array}{l}\text { Performance } \\
\text { efficacy }\end{array}$ & $\begin{array}{c}\text { time to complete } \\
\text { (minutes) }\end{array}$ & $\begin{array}{l}\text { nature-based exercisers } \\
\text { took less time to complete } \\
\text { trial }\end{array}$ \\
\hline & & & & & & & $\begin{array}{l}\text { Performance } \\
\text { efficiency }\end{array}$ & Heart Rate $(\mathrm{bpm})$ & $\begin{array}{l}\text { Lower Heart rate for indoor } \\
\text { exercisers }\end{array}$ \\
\hline & & & & & & & $\begin{array}{l}\text { Performance } \\
\text { efficiency }\end{array}$ & $\begin{array}{c}\text { Rating of Perceived } \\
\text { Exertion }\end{array}$ & no differences \\
\hline & & & & & & & $\begin{array}{l}\text { Performance } \\
\text { efficiency }\end{array}$ & $\begin{array}{c}\text { Tammen } \\
\text { attentional focus } \\
\text { scale }\end{array}$ & no differences \\
\hline \multirow{3}{*}{$\begin{array}{c}\text { Mugele } \mathrm{H} \text {, Plummer A, } \\
\text { Baritello O, Towe M, Brecht P, } \\
\text { Mayer F. Accuracy of training } \\
\text { recommendations based on a } \\
\text { treadmill multistage } \\
\text { incremental exercise test. PLoS } \\
\text { One. 2018;13:e0204696. }\end{array}$} & \multirow{3}{*}{$\begin{array}{l}\text { non } \\
\text { randomised } \\
\text { crossover } \\
\text { controlled trial }\end{array}$} & \multirow{3}{*}{ acute } & \multirow{3}{*}{$\begin{array}{c}15 \text { experienced runners, } 7 \\
\text { females, age } 30(27-34) \\
\text { years old }\end{array}$} & \multirow{3}{*}{$\begin{array}{l}\text { incremental running } \\
\text { exercise testing }\end{array}$} & \multirow{3}{*}{$\begin{array}{l}\text { a } 400 \mathrm{~m} \text { tartan athletic } \\
\text { outdoor running track }\end{array}$} & \multirow{3}{*}{ Indoor laboratory } & $\begin{array}{c}\text { Performance } \\
\text { efficacy }\end{array}$ & distance ran & $\begin{array}{l}\text { greater running distance } \\
\text { covered in nature-based } \\
\text { exercisers }\end{array}$ \\
\hline & & & & & & & $\begin{array}{l}\text { Performance } \\
\text { efficiency }\end{array}$ & $\begin{array}{c}\text { Rating of Perceived } \\
\text { Exertion }\end{array}$ & $\begin{array}{l}\text { lower perceived exertion in } \\
\text { nature-based exercise }\end{array}$ \\
\hline & & & & & & & & & \\
\hline
\end{tabular}




\begin{tabular}{|c|c|c|c|c|c|c|c|c|c|}
\hline $\begin{array}{l}\text { Natera AO, Jennings J, Oakley } \\
\text { AJ, Jones TW. Influence of } \\
\text { environmental conditions on } \\
\text { performance and heart rate } \\
\text { responses to the } 30-15 \\
\text { incremental fitness test in } \\
\text { rugby union athletes. J } \\
\text { Strength Cond Res. } \\
2019 ; 33: 486-91 .\end{array}$ & 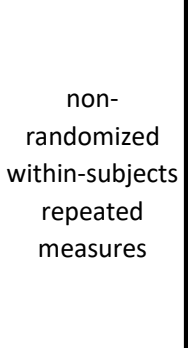 & acute & $\begin{array}{l}8 \text { male highly trained Rugby } \\
\text { Union players, age } 28.1 \pm 1.5\end{array}$ & $\begin{array}{c}\text { incremental } 30 \text {-second } \\
\text { shuttle runs over a } 40-\mathrm{m} \\
\text { distance, interspersed with } \\
15 \text {-second walking recovery } \\
\text { period }\end{array}$ & $\begin{array}{c}\text { outdoors on a grass pitch } \\
\text { in highheat }\end{array}$ & $\begin{array}{l}\text { indoors on a } 4 \mathrm{G} \text { artificial } \\
\text { pitch }\end{array}$ & $\begin{array}{l}\text { Performance } \\
\text { efficiency }\end{array}$ & Heart Rate (bpm) & $\begin{array}{l}\text { greater speed in indoor } \\
\text { exercisers }\end{array}$ \\
\hline \multirow{5}{*}{$\begin{array}{l}\text { Navalta JW, Bodell NG, Tanner } \\
\text { EA, Aguilar CD, Radzak KN. } \\
\text { Effect of exercise in a desert } \\
\text { environment on physiological } \\
\text { and subjective measures. Int J } \\
\text { Environ Health Res. } \\
\text { 2021;31:121-31. }\end{array}$} & \multirow{5}{*}{$\begin{array}{c}\text { non- } \\
\text { randomized } \\
\text { within-subjects } \\
\text { repeated } \\
\text { measures }\end{array}$} & \multirow{5}{*}{ acute } & \multirow{5}{*}{$\begin{array}{l}10 \text { volunteers (age } 29.2 \pm \\
7.3), 7 \text { female }\end{array}$} & \multirow{5}{*}{$\begin{array}{c}\text { 30-min self-paced walking in } \\
\text { nature or indoors (no } \\
\text { treadmill) }\end{array}$} & \multirow{5}{*}{\begin{tabular}{|c|} 
Mt. Charleston \\
Bristlecone Trail, which is \\
part ofthe Spring \\
Mountains National \\
Recreation Area
\end{tabular}} & \multirow{5}{*}{$\begin{array}{l}\text { Exercise Physiology } \\
\text { laboratory }\end{array}$} & $\begin{array}{l}\text { Performance } \\
\text { efficiency }\end{array}$ & Heart Rate (bpm) & no differences \\
\hline & & & & & & & $\begin{array}{l}\text { Performance } \\
\text { efficacy }\end{array}$ & miles ran & no differences \\
\hline & & & & & & & Well-being & $\begin{array}{l}\text { perceived Stress } \\
\text { Scale }\end{array}$ & no differences \\
\hline & & & & & & & Well-being & Comfort & $\begin{array}{l}\text { greater comfort in nature- } \\
\text { based exercise }\end{array}$ \\
\hline & & & & & & & Well-being & calm & $\begin{array}{l}\text { greater calm in nature- } \\
\text { based exercise }\end{array}$ \\
\hline
\end{tabular}




\begin{tabular}{|c|c|c|c|c|c|c|c|c|c|}
\hline \multirow{3}{*}{$\begin{array}{l}\text { Niedermeier M, Grafetstaetter } \\
\text { C, Hartl A, Kopp M. A } \\
\text { Randomized Crossover Trial on } \\
\text { Acute Stress-Related } \\
\text { Physiological Responses to } \\
\text { Mountain Hiking. Int J Environ } \\
\text { Res Public Health. 2017;14. }\end{array}$} & \multirow{3}{*}{$\begin{array}{c}\text { randomized } \\
\text { within-subjects } \\
\text { repeated } \\
\text { measures }\end{array}$} & \multirow{3}{*}{ acute } & \multirow{3}{*}{$\begin{array}{c}42 \text { healthy adults (age } 32 \pm \\
12 \text { ), } 20 \text { female }\end{array}$} & \multirow{3}{*}{$\begin{array}{l}6 \mathrm{~km} \text { uphill hiking } \\
\text { approximately } 90 \text { min and } \\
\text { downhill hiking around } 70 \\
\text { min. Walking uphill on } \\
\text { indoor treadmill followed by } \\
\text { level, aproximately same } \\
\text { time periods and same } \\
\text { average speed }\end{array}$} & \multirow{3}{*}{$\begin{array}{l}\text { single trails and forest } \\
\text { roads to a mountain hut } \\
(1500 \mathrm{~m}) \text { with a view of } \\
\text { the mountainous region }\end{array}$} & \multirow{3}{*}{ indoor fitness center } & Performance & Heart Rate (bpm) & no differences \\
\hline & & & & & & & $\begin{array}{c}\text { Performance } \\
\text { efficacy }\end{array}$ & Total power & no differences \\
\hline & & & & & & & $\begin{array}{c}\text { Performance } \\
\text { efficiency }\end{array}$ & $\mid \begin{array}{c}\text { Rating of Perceived } \\
\text { Exertion }\end{array}$ & $\begin{array}{l}\text { lower perceived exertion } \\
\text { for nature-based exercisers }\end{array}$ \\
\hline \multirow{4}{*}{$\begin{array}{l}\text { Niedermeier M, Einwanger J, } \\
\text { Hartl A, Kopp M. Affective } \\
\text { responses in mountain hiking- } \\
\text { A randomized crossover trial } \\
\text { focusing on differences } \\
\text { between indoor and outdoor } \\
\text { activity. PLoS One. } \\
\text { 2017;12:e0177719. }\end{array}$} & \multirow{4}{*}{$\begin{array}{c}\text { randomized } \\
\text { within-subjects } \\
\text { repeated } \\
\text { measures }\end{array}$} & \multirow{4}{*}{ acute } & \multirow{4}{*}{$\begin{array}{c}42 \text { healthy adults (age } 32 \pm \\
12 \text { ), } 20 \text { female }\end{array}$} & \multirow{4}{*}{$\begin{array}{l}6 \mathrm{~km} \text { uphill hiking } \\
\text { approximately } 90 \text { min and } \\
\text { downhill hiking around } 70 \\
\text { min. Walking uphill on } \\
\text { indoor treadmill followed by } \\
\text { level, aproximately same } \\
\text { time periods and same } \\
\text { average speed }\end{array}$} & \multirow{4}{*}{$\begin{array}{l}\text { single trails and forest } \\
\text { roads to a mountain hut } \\
(1500 \mathrm{~m}) \text { with a view of } \\
\text { the mountainous region }\end{array}$} & \multirow{4}{*}{ indoor fitness center } & Well-being & Feeling Scale & $\begin{array}{l}\text { greater positive affect for } \\
\text { nature-based exercisers }\end{array}$ \\
\hline & & & & & & & $\begin{array}{l}\text { Performance } \\
\text { efficiency }\end{array}$ & Felt Arousal Scale & no differences \\
\hline & & & & & & & $\begin{array}{c}\text { Performance } \\
\text { efficiency }\end{array}$ & $\begin{array}{c}\text { activation (Mood } \\
\text { Survey Scale) }\end{array}$ & $\begin{array}{l}\text { greater activation for } \\
\text { nature-based exercisers }\end{array}$ \\
\hline & & & & & & & $\begin{array}{l}\text { Performance } \\
\text { efficiency }\end{array}$ & $\begin{array}{l}\text { fatigue (Mood } \\
\text { Survey Scale) }\end{array}$ & $\begin{array}{l}\text { lower fatigue for nature- } \\
\text { based exercisers }\end{array}$ \\
\hline
\end{tabular}




\begin{tabular}{|c|c|c|c|c|c|c|c|c|c|}
\hline & & & & & & & Well-being & $\begin{array}{c}\text { State Trait Anxiety } \\
\text { Inventory }\end{array}$ & no differences \\
\hline & & & & & & & $\begin{array}{l}\text { Performance } \\
\text { efficiency }\end{array}$ & $\left|\begin{array}{c}\text { Rating of Perceived } \\
\text { Exertion }\end{array}\right|$ & no differences \\
\hline & & & & & & & $\begin{array}{c}\text { Performance } \\
\text { efficiency }\end{array}$ & $\begin{array}{l}\text { Heart Rate (\% } \\
\text { Hrmax) }\end{array}$ & $\begin{array}{l}\text { ower Heart rate for indoor } \\
\text { exercisers }\end{array}$ \\
\hline \multirow{3}{*}{\begin{tabular}{|c} 
Olafsdottir G, Cloke P, Schulz \\
A, van Dyck Z, Eysteinsson T, \\
Thorleifsdottir B, et al. Health \\
Benefits of Walking in Nature: \\
A Randomized Controlled \\
Study Under Conditions of Real \\
Life Stress. Environ Behav \\
[Internet]. 2020;52:248-74.
\end{tabular}} & \multirow{3}{*}{$\begin{array}{c}\text { randomized } \\
\text { independent } \\
\text { groups }\end{array}$} & \multirow{3}{*}{ acute } & \multirow{3}{*}{$\begin{array}{l}67 \text { eligible participants, age } \\
24.4 \pm 2.6 \text { years, gender } \mathrm{n} / \mathrm{a}\end{array}$} & \multirow{3}{*}{$\begin{array}{l}\text { 40-min walk on treadmill } \\
\text { indoors or in nature } \\
\text { (instructed to keep a } \\
\text { comfortable walking speed) }\end{array}$} & \multirow{3}{*}{$\begin{array}{c}32 \mathrm{~km} 2 \text { are covered with } \\
\text { woodland of } 26 \text { different } \\
\text { species predominated } \\
\text { with the Sitka Spruce } \\
\text { (Picea sitchensis). }\end{array}$} & \multirow{3}{*}{$\begin{array}{l}\text { a gym selected because of } \\
\text { its easily accessible } \\
\text { location, modern man- } \\
\text { made infrastructure }\end{array}$} & $\begin{array}{l}\text { Performance } \\
\text { efficiency }\end{array}$ & $\begin{array}{l}\text { Heart Rate and } \\
\text { Heart Rate } \\
\text { Variability }\end{array}$ & no differences \\
\hline & & & & & & & Well-being & \begin{tabular}{c|} 
negative affect \\
(Positive and \\
Negative Affect \\
Scale)
\end{tabular} & no differences \\
\hline & & & & & & & Well-being & $\begin{array}{l}\text { positive affect } \\
\text { (Positive and } \\
\text { Negative Affect } \\
\text { Scale) }\end{array}$ & $\begin{array}{l}\text { greater positive affect in } \\
\text { nature-based exercisers }\end{array}$ \\
\hline & & & & & & & Well-being & $\begin{array}{l}\text { Rosenberg Self- } \\
\text { esteem Scale }\end{array}$ & $\begin{array}{l}\text { greater self-esteem in } \\
\text { nature-based exercisers }\end{array}$ \\
\hline & & & & & & & & & \\
\hline
\end{tabular}




\begin{tabular}{|c|c|c|c|c|c|c|c|c|c|}
\hline \multirow[t]{2}{*}{$\begin{array}{l}\text { Peacock J, Hine R, Pretty JN. } \\
\text { The mental health benefits of } \\
\text { green exercise activities and } \\
\text { green care. Mind Week Rep. } \\
\text { 2007;1-18. }\end{array}$} & \multirow[t]{2}{*}{$\begin{array}{c}\text { non- } \\
\text { randomized } \\
\text { within-subjects } \\
\text { repeated } \\
\text { measures }\end{array}$} & \multirow[t]{2}{*}{ acute } & \multirow[t]{2}{*}{$\begin{array}{c}20 \text { members of local mind } \\
\text { associations, } 13 \text { female; } 47 \% \\
\text { were between } 31-50 \text { yo, and } \\
53 \% \text { were between } 51-70 \text { yo }\end{array}$} & \multirow[t]{2}{*}{$\begin{array}{c}\text { 1h group walk (self-selected } \\
\text { intensity) }\end{array}$} & \multirow[t]{2}{*}{$\begin{array}{l}\text { Belhus Woods Country } \\
\text { Park, which has a diverse } \\
\text { landscape of woodlands, } \\
\text { grasslands and lakes }\end{array}$} & \multirow[t]{2}{*}{ Lakeside shopping centre } & Well-being & $\begin{array}{l}\text { anger, confusion, } \\
\text { depression, } \\
\text { tension (Profile of } \\
\text { Mood States) }\end{array}$ & $\begin{array}{l}\text { lower anger, confusion, } \\
\text { depression, and tension in } \\
\text { nature-based exercisers }\end{array}$ \\
\hline & & & & & & & $\begin{array}{l}\text { Performance } \\
\text { efficiency }\end{array}$ & $\begin{array}{c}\text { fatigue, vigor } \\
\text { (Profile of Mood } \\
\text { States) }\end{array}$ & no differences \\
\hline $\begin{array}{l}\text { Plante TG, Gores C, Brecht C, } \\
\text { Carrow J, Imbs A, Willemsen E. } \\
\text { Does exercise environment } \\
\text { enhance the psychological } \\
\text { benefits of exercise for } \\
\text { women? Int J Stress Manag } \\
\text { [Internet]. 2007 [cited } 2020 \\
\text { Jan 30];14:88-98. }\end{array}$ & $\begin{array}{c}\text { randomized } \\
\text { independent } \\
\text { groups }\end{array}$ & acute & $\begin{array}{l}88 \text { female undergraduate } \\
\text { students, age } 19.3 \pm 0.9\end{array}$ & $\begin{array}{c}20 \text { minute walk alone or } \\
\text { acompanied in nature or on } \\
\text { indoor treadmill (keep their } \\
\text { heart rate between } 120 \text { and } \\
140 \text { beats per minute. } \\
\text { Participants walked for } 20 \\
\text { min at a moderate speed } \\
\text { (i.e., } 60 \% \text { and } 70 \% \text { of their } \\
\text { maximum heart rate) }\end{array}$ & $\begin{array}{l}\text { prescribed route on the } \\
\text { Santa Clara University } \\
\text { campus }\end{array}$ & university fitness facility & $\begin{array}{c}\begin{array}{c}\text { Performance } \\
\text { efficiency }\end{array} \\
\text { Well-being }\end{array}$ & $\begin{array}{c}\text { Activation- } \\
\text { Deactivation } \\
\text { Adjective Checklist }\end{array}$ & no differences \\
\hline \multirow{2}{*}{$\begin{array}{c}\text { Plante TG, Aldridge A, Su D, } \\
\text { Bogdan R, Belo M, Kahn K. } \\
\text { Does Virtual Reality Enhance } \\
\text { the Management of Stress } \\
\text { When Paired With Exercise? } \\
\text { An Exploratory Study. Int J } \\
\text { Stress Manag. } \\
\text { 2003;10:203-16. }\end{array}$} & \multirow{2}{*}{$\begin{array}{c}\text { randomized } \\
\text { independent } \\
\text { groups }\end{array}$} & \multirow{2}{*}{$\begin{array}{l}\text { acute (nature } \\
\text { simulation) }\end{array}$} & \multirow{2}{*}{$\begin{array}{l}52 \text { male introductory } \\
\text { psychology students (age } \\
\text { n/a); } 102 \text { female } \\
\text { introductory psychology } \\
\text { students (age n/a) }\end{array}$} & \multirow{2}{*}{$\begin{array}{l}20 \text { minute brisk walk } \\
\text { (intensity about } 3 \text { miles per } \\
\text { hour) outdoor or on a } \\
\text { treadmill indoor }\end{array}$} & \multirow{2}{*}{$\begin{array}{l}\text { predetermined route } \\
\text { around campus }\end{array}$} & \multirow{2}{*}{ laboratory } & Well-being & $\begin{array}{l}\text { Calmness, Tension } \\
\text { (Activation- } \\
\text { Deactivation } \\
\text { Adjective Checklist) }\end{array}$ & no differences \\
\hline & & & & & & & $\begin{array}{c}\text { Performance } \\
\text { efficiency }\end{array}$ & $\begin{array}{l}\text { energy (Activation- } \\
\text { Deactivation } \\
\text { Adjective Checklist) }\end{array}$ & $\begin{array}{l}\text { greater energy in nature- } \\
\text { based exerciseres, but only } \\
\text { in female participants }\end{array}$ \\
\hline
\end{tabular}




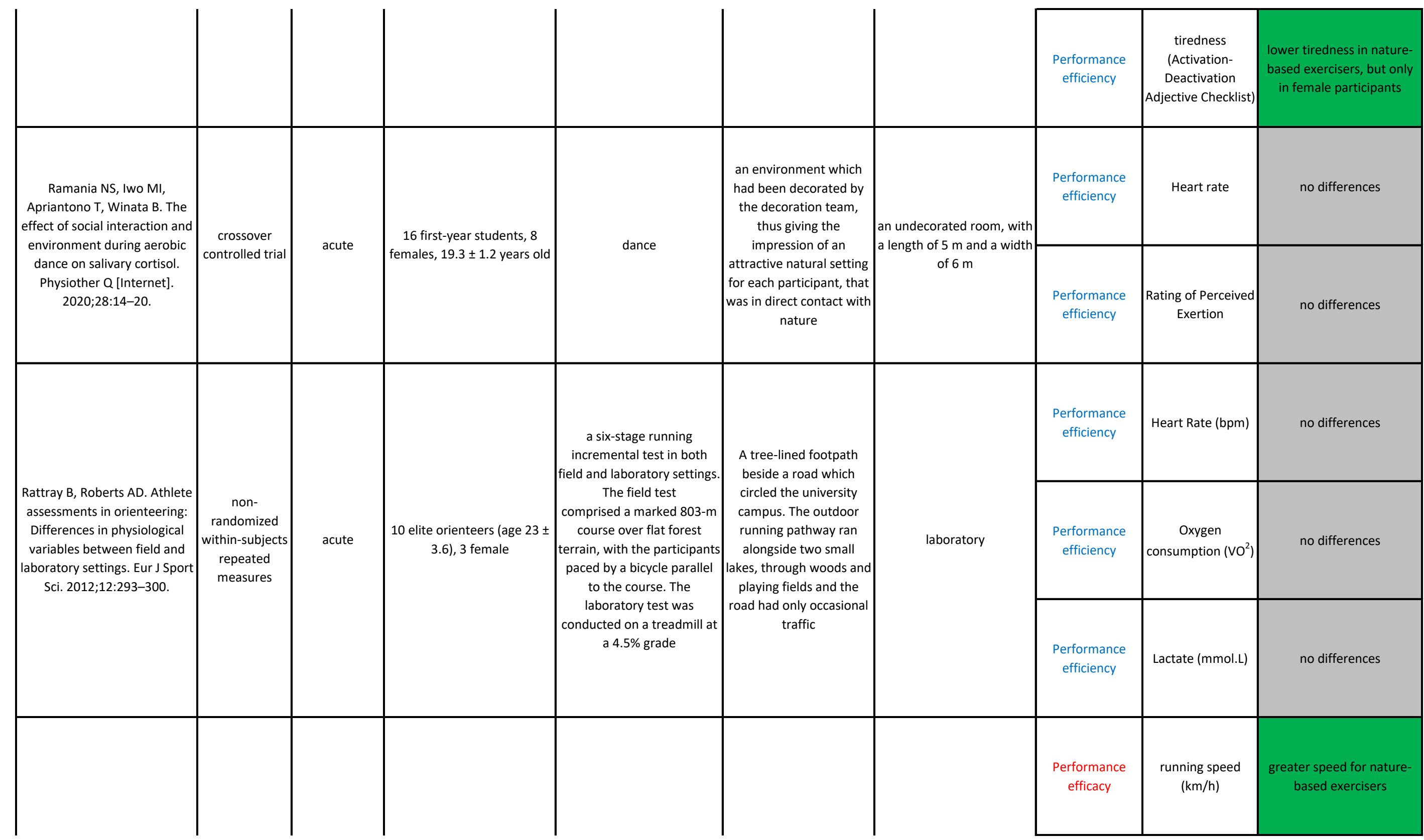


Reich AH, Queathem EJ. Setting, Age, and Intensity

Influence Responses to

Exercise in Young Endurance

Runners. Percept Mot Skills

[Internet]. 2020;127:533-54. randomized

within-subjects

repeated

measures
48 male club runners (age

middleschool: $13 \pm 1.2$; high

school: $16.7 \pm 1.3$; college:

$20 \pm 1.1$ three 6-minute runs in

indoor treadmill and in

outdoor trail with increasing

intensity each run,

according to three levels of

perceived exertion $(10,12$ and 16) dirt path through a forest beside a lake standard motor-driven treadmill in a college fitness center

-

Rogerson M, Barton JL. Effects of the Visual Exercise Environments on Cognitive Directed Attention, Energy Expenditure and Perceived Exertion. Int J Environ Res Public Health [Internet]. 2015;12:7321-36. balanced repeated measures
12 healthy adult students acute (nature and staff at the University of simulation) Essex, 6 female, age $27.8 \pm$

5.5 15-min bout of running xercise on the treadmill at $60 \%$ V02peaklnt, followed by $85 \%$ VO2peakInt until voluntary exhaustion while watching nature video, or blank screen
In the nature condition, the video consisted of scenes extracted from "Evening Run through Endless Forest"

\begin{tabular}{|c|c|c|}
\hline $\begin{array}{l}\text { Performance } \\
\text { efficiency }\end{array}$ & $\begin{array}{l}\text { Heart Rate / Heart } \\
\text { Rate max }\end{array}$ & $\begin{array}{c}\text { lower Heart rate for indoor } \\
\text { exercisers }\end{array}$ \\
\hline $\begin{array}{l}\text { Performance } \\
\text { efficiency }\end{array}$ & $\begin{array}{l}\text { Thoughts During } \\
\text { Running Scale }\end{array}$ & $\begin{array}{l}\text { more associative thoughts } \\
\text { for nature-based exercisers }\end{array}$ \\
\hline $\begin{array}{l}\text { Performance } \\
\text { efficiency } \\
\text { Well-being }\end{array}$ & $\begin{array}{l}\text { Physical Activity } \\
\text { Affect Scale }\end{array}$ & no differences \\
\hline $\begin{array}{l}\text { Performance } \\
\text { efficacy }\end{array}$ & $\begin{array}{c}\text { time to exhaustion } \\
\text { (seconds) }\end{array}$ & no differences \\
\hline $\begin{array}{l}\text { Performance } \\
\text { efficiency }\end{array}$ & \begin{tabular}{c|} 
Energy \\
expenditure (kcal)
\end{tabular} & no differences \\
\hline $\begin{array}{l}\text { Performance } \\
\text { efficiency }\end{array}$ & Heart Rate $(\mathrm{bpm})$ & no differences \\
\hline $\begin{array}{l}\text { Performance } \\
\text { efficiency }\end{array}$ & $\begin{array}{l}\text { backwards digit } \\
\text { span task }\end{array}$ & $\begin{array}{l}\text { greater directed attention } \\
\text { in nature-based exercisers }\end{array}$ \\
\hline
\end{tabular}




\begin{tabular}{|c|c|c|c|c|c|c|c|c|c|}
\hline & & & & & & & $\begin{array}{l}\text { Performance } \\
\text { efficiency }\end{array}$ & $\begin{array}{c}\text { Rating of Perceived } \\
\text { Exertion }\end{array}$ & no differences \\
\hline \multirow{4}{*}{$\begin{array}{l}\text { Rogerson M, Gladwell VF, } \\
\text { Gallagher DJ, Barton JL. } \\
\text { Influences of Green Outdoors } \\
\text { versus Indoors Environmental } \\
\text { Settings on Psychological and } \\
\text { Social Outcomes of Controlled } \\
\text { Exercise. Int J Environ Res } \\
\text { Public Health. 2016;13:363. }\end{array}$} & \multirow{4}{*}{$\begin{array}{l}\text { randomized } \\
\text { counter- } \\
\text { balanced } \\
\text { repeated } \\
\text { measures }\end{array}$} & \multirow{4}{*}{ acute } & \multirow{4}{*}{$\begin{array}{c}24 \text { university staff and } \\
\text { students (10) and and } \\
\text { general public from Essex } \\
\text { (13), } 19 \text { female, age } 35.1 \pm \\
20.1\end{array}$} & \multirow{4}{*}{$\begin{array}{c}15 \text { minute cycle ergometer } \\
\text { indoors and outdoors (50\% } \\
\text { Heart rate reserve) }\end{array}$} & \multirow{4}{*}{$\begin{array}{l}\text { University of Essex sports } \\
\quad \text { fields, } \\
\text { which is a large area of } \\
\text { largely level gradient, } \\
\text { maintained grassland, } \\
\text { lined and partly } \\
\text { interspersed } \\
\text { with trees }\end{array}$} & \multirow{4}{*}{$\begin{array}{c}\text { laboratory setting, } \\
\text { whereby participants' view } \\
\text { was of a white painted } \\
\text { brick wall. The dimensions } \\
\text { of the laboratory were } 8.3 \\
\mathrm{~m} \times 4,9 \mathrm{~m}\end{array}$} & $\begin{array}{c}\text { Performance } \\
\text { efficiency } \\
\text { Well-being }\end{array}$ & \begin{tabular}{|c|} 
total mood \\
disturbance \\
(Profile of Mood \\
States)
\end{tabular} & no differences \\
\hline & & & & & & & $\begin{array}{c}\text { Performance } \\
\text { efficiency }\end{array}$ & $\begin{array}{c}\text { Rating of Perceived } \\
\text { Exertion }\end{array}$ & no differences \\
\hline & & & & & & & $\begin{array}{l}\text { Performance } \\
\text { efficiency }\end{array}$ & $\begin{array}{l}\text { Backwards Digit } \\
\text { Span Test }\end{array}$ & no differences \\
\hline & & & & & & & $\begin{array}{l}\text { Performance } \\
\text { efficiency } \\
\text { Well-being }\end{array}$ & $\begin{array}{l}\text { Profile of Mood } \\
\text { States }\end{array}$ & no differences \\
\hline \multirow{2}{*}{$\begin{array}{l}\text { Ross R, ALDuhishy A, Gonzalez- } \\
\text { Haro C. Validation of the } \\
\text { cosmed K4B2 portable } \\
\text { metabolic system during } \\
\text { running outdoors. I strength } \\
\text { Cond Res. 2020;34:124-33. }\end{array}$} & \multirow{2}{*}{$\begin{array}{c}\text { randomized } \\
\text { within-subjects } \\
\text { repeated } \\
\text { measures }\end{array}$} & \multirow{2}{*}{ acute } & \multirow{2}{*}{$\begin{array}{l}19 \text { male endurance-trained } \\
\text { volunteers (age } 22.9 \pm 1 \text { ) }\end{array}$} & \multirow{2}{*}{$\begin{array}{c}\text { run } 8 \mathrm{~km} / \mathrm{h} \text { with increments } \\
\text { of } 2 \mathrm{~km} / \mathrm{h} \text { every } 3 \text { minutes } \\
\text { until exhaustion }\end{array}$} & \multirow{2}{*}{\begin{tabular}{|} 
400-m concrete running \\
track (Bellahouston Park, \\
Glasgow, United Kingdom
\end{tabular}} & \multirow{2}{*}{$\begin{array}{l}\text { treadmill in an ambient } \\
\text { temperature controlled } \\
\text { room }\end{array}$} & $\begin{array}{l}\text { Performance } \\
\text { efficiency }\end{array}$ & Heart Rate (bpm) & $\begin{array}{l}\text { lower Heart rate for nature- } \\
\text { based exercisers }\end{array}$ \\
\hline & & & & & & & $\begin{array}{l}\text { Performance } \\
\text { efficiency }\end{array}$ & $\mid$\begin{tabular}{|c|}
$\mathrm{VO} 2, \mathrm{VCO}_{2}, \mathrm{~F}_{\mathrm{E}} \mathrm{O}_{2}$ \\
$\mathrm{~F}_{\mathrm{E}} \mathrm{CO}_{2}, \mathrm{~V}_{\mathrm{E}} \mathrm{RER}, \mathrm{HR}$
\end{tabular} & $\begin{array}{l}\text { lower respiratory output for } \\
\text { nature-based exercisers }\end{array}$ \\
\hline
\end{tabular}




\begin{tabular}{|c|c|c|c|c|c|c|c|c|c|}
\hline & & & & & & & $\begin{array}{l}\text { Performance } \\
\text { efficacy }\end{array}$ & $\begin{array}{l}\text { running speed } \\
(\mathrm{km} / \mathrm{h})\end{array}$ & no differences \\
\hline $\begin{array}{l}\text { Ryan RM, Weinstein N, } \\
\text { Bernstein J, Brown KW, } \\
\text { Mistretta L, Gagné M. } \\
\text { Vitalizing effects of being } \\
\text { outdoors and in nature. J } \\
\text { Environ Psychol. } \\
\text { 2010;30:159-68. }\end{array}$ & $\begin{array}{c}\text { randomized } \\
\text { independent } \\
\text { groups }\end{array}$ & acute & $\begin{array}{l}80 \text { undergraduates, } 66 \\
\text { women }(82.5 \%) \text {, age } 20\end{array}$ & $\begin{array}{l}\text { short walk for } 15 \mathrm{~min} \text {; both } \\
\text { outdoor and } \\
\text { indoorwalkswere the same } \\
\text { length.Experimenters were } \\
\text { instructed to walk at the } \\
\text { same speed in both } \\
\text { conditions }\end{array}$ & $\begin{array}{c}\text { a largely tree-lined } \\
\text { footpath along a river } \\
\text { that runs parallel to the } \\
\text { university campus }\end{array}$ & $\begin{array}{c}\text { underground hallways and } \\
\text { tunnels that were devoid of } \\
\text { living things, although } \\
\text { there were many objects, } \\
\text { posters, physical changes, } \\
\text { and colors present }\end{array}$ & Well-being & $\begin{array}{c}\text { Subjective Vitality } \\
\text { Scale }\end{array}$ & $\begin{array}{l}\text { greater vitality in nature- } \\
\text { based exercisers }\end{array}$ \\
\hline \multirow{4}{*}{$\begin{array}{l}\text { Shin Y-KK, Kim DJ, Jung-Choi K, } \\
\text { Son Y-J, Koo J-WW, Min J-AA, } \\
\text { et al. Differences of } \\
\text { psychological effects between } \\
\text { meditative and athletic } \\
\text { walking in a forest and } \\
\text { gymnasium. Scand J Jor Res. } \\
\text { 2013;28:64-72. }\end{array}$} & \multirow{4}{*}{$\begin{array}{l}\text { randomized } \\
\text { independent } \\
\text { groups }\end{array}$} & \multirow{4}{*}{ acute } & \multirow{4}{*}{$\begin{array}{l}139 \text { community-dwelling } \\
\text { females (age } 20.4 \pm 1.5 \text { ) }\end{array}$} & \multirow{4}{*}{$\begin{array}{l}\text { meditative walk ( } 40-60 \% \text { of } \\
\text { their age-predicted } \\
\text { maximum heart rates) and } \\
\text { athletic walk (60-85\% of the } \\
\text { same heart rates) indoor } \\
\text { and outdoor. All subjects } \\
\text { walked for } 35 \mathrm{~min} \text {, then } \\
\text { rested for } 10 \mathrm{~min} \text {, and } \\
\text { repeated the sequence once } \\
\text { more. Each of the four } \\
\text { walking program types took } \\
90 \mathrm{~min}\end{array}$} & \multirow{4}{*}{$\begin{array}{c}\text { Jocheon- } \\
\text { Hamdeok Gotjawal } \\
\text { Forest is a natural forest } \\
\text { located } \\
\text { on the middle slopes of } \\
\text { Halla Mountain, Jeju } \\
\text { Island, } \\
\text { off the southwestern } \\
\text { coast of South Korea. }\end{array}$} & \multirow{4}{*}{\begin{tabular}{|c|} 
gymnasium of Shinseong \\
Girl's High School, Jeju City, \\
South Korea. The \\
gymnasium has a 100-m \\
indoor track. All \\
activities in the gymnasium \\
groups were performed \\
indoors of the gymnasium
\end{tabular}} & $\begin{array}{l}\text { Performance } \\
\text { efficacy }\end{array}$ & \begin{tabular}{|c} 
distance covered \\
$(\mathrm{km})$
\end{tabular} & no differences \\
\hline & & & & & & & $\begin{array}{l}\text { Performance } \\
\text { efficiency }\end{array}$ & $\%$ Heart Rate max & no differences \\
\hline & & & & & & & Well-being & $\begin{array}{c}\text { State Trait Anxiety } \\
\text { Inventory- } \mathrm{X} 1\end{array}$ & no differences \\
\hline & & & & & & & Well-being & $\begin{array}{l}\text { Rosenberg Self- } \\
\text { esteem Scale }\end{array}$ & no differences \\
\hline
\end{tabular}




\begin{tabular}{|c|c|c|c|c|c|c|c|c|c|}
\hline & & & & & & & Well-being & $\begin{array}{c}\text { Happiness Index } \\
\text { for Koreans) }\end{array}$ & $\begin{array}{l}\text { greater happiness in nature } \\
\text { based exercisers, but only } \\
\text { meditative walk exercisers }\end{array}$ \\
\hline \multirow{3}{*}{$\begin{array}{l}\text { Slapsinskaite A, Garcia S, } \\
\text { Razon S, Balague N, Hristovski } \\
\text { R, Tenenbaum G. Cycling } \\
\text { outdoors facilitates external } \\
\text { thoughts and endurance. } \\
\text { Psychol Sport Exerc. } \\
\text { 2016;27:78-84. }\end{array}$} & \multirow{3}{*}{$\begin{array}{l}\text { randomized } \\
\text { counter- } \\
\text { balanced } \\
\text { repeated } \\
\text { measures }\end{array}$} & \multirow{3}{*}{ acute } & \multirow{3}{*}{$\begin{array}{c}13 \text { Caucasian physical } \\
\text { education students, } 3 \\
\text { female, age } 21.7, \operatorname{SEM}=0.8 \text { ) }\end{array}$} & \multirow{3}{*}{$\begin{array}{l}\text { cycling. Initial load of } 20 \mathrm{~W} \\
\text { (for females) and } 25 \mathrm{~W} \text { (for } \\
\text { males). The load was } \\
\text { increased until reaching the } \\
\text { RPE }=15 \text {. The test was } \\
\text { terminated when } \\
\text { participants could no longer } \\
\text { maintain the required pace } \\
(70 \text { rpm) for } 5 \text { consecutive } \\
\text { seconds. }\end{array}$} & \multirow{3}{*}{$\begin{array}{c}\text { participants cycled in an } \\
\text { urban setting near a } \\
\text { roadway and a state park } \\
\text { with sparse vegetation } \\
\text { and some mountain } \\
\text { scenery }\end{array}$} & \multirow{3}{*}{$\begin{array}{l}\text { human performance } \\
\text { laboratory. environmental } \\
\text { conditions within the } \\
\text { laboratory were constant } \\
\text { with the cycle ergometer } \\
\text { placed in front of the wal }\end{array}$} & $\begin{array}{c}\text { Performance } \\
\text { efficacy }\end{array}$ & $\begin{array}{c}\text { cycling endurance } \\
(\mathrm{min})\end{array}$ & $\begin{array}{l}\text { greater cycling endurance } \\
\text { in nature-based exercisers }\end{array}$ \\
\hline & & & & & & & $\begin{array}{l}\text { Performance } \\
\text { efficiency }\end{array}$ & $\left|\begin{array}{c}\text { thoughts related to } \\
\text { the task }\end{array}\right|$ & $\begin{array}{l}\text { more thoughts related to } \\
\text { the task in nature-based } \\
\text { exercisers }\end{array}$ \\
\hline & & & & & & & $\begin{array}{l}\text { Performance } \\
\text { efficiency }\end{array}$ & $\begin{array}{c}\text { thoughts unrelated } \\
\text { to the task }\end{array}$ & $\begin{array}{c}\text { more thoughts unrelated } \\
\text { to the task in nature-based } \\
\text { exercisers }\end{array}$ \\
\hline \multirow{3}{*}{$\begin{array}{l}\text { Teas J, Hurley T, Ghumare S, } \\
\text { Ogoussan K. Walking outside } \\
\text { Improves Mood for Healthy } \\
\text { Postmenopausal Women. Clin } \\
\text { Med Oncol. 2007;1:CMO.S343. }\end{array}$} & \multirow{3}{*}{$\begin{array}{c}\text { non- } \\
\text { randomized } \\
\text { within-subjects } \\
\text { repeated } \\
\text { measures }\end{array}$} & \multirow{3}{*}{ acute } & \multirow{3}{*}{$\begin{array}{c}19 \text { post-menopausal women, } \\
\text { age } 58 \pm 4\end{array}$} & \multirow{3}{*}{$\begin{array}{l}1 \text { hour walk on treadmill } \\
\text { indoors and } 1 \text { hour walk } \\
\text { outdoors; self-selected } \\
\text { intensity }\end{array}$} & \multirow{3}{*}{$\begin{array}{l}\text { The campus walk was } \\
\text { esthetically pleasing, } \\
\text { following brick pathways } \\
\text { lined with fl owering } \\
\text { plants and large trees }\end{array}$} & \multirow{3}{*}{$\begin{array}{l}\text { The gym lab was located in } \\
\text { the university gym, and } \\
\text { provided an environment } \\
\text { similar to that found in a } \\
\text { commercial gym }\end{array}$} & $\begin{array}{l}\text { Performance } \\
\text { efficacy }\end{array}$ & $\begin{array}{l}\text { Walking speed } \\
(\mathrm{km} / \mathrm{h})\end{array}$ & no differences \\
\hline & & & & & & & Well-being & $\begin{array}{l}\text { Positive affect } \\
\text { scale }\end{array}$ & $\begin{array}{l}\text { greater positive affect for } \\
\text { nature-based exercisers }\end{array}$ \\
\hline & & & & & & & Well-being & $\begin{array}{l}\text { negative affect } \\
\text { scale }\end{array}$ & $\begin{array}{l}\text { lower negative affect for } \\
\text { nature-based exercisers }\end{array}$ \\
\hline
\end{tabular}




\begin{tabular}{|c|c|c|c|c|c|c|c|c|c|}
\hline \multirow{5}{*}{$\begin{array}{c}\text { Turner TL, Stevinson C. } \\
\text { Affective outcomes during and } \\
\text { after high-intensity exercise in } \\
\text { outdoor green and indoor gym } \\
\text { settings. Int J Environ Health } \\
\text { Res [Internet]. Taylor \& } \\
\text { Francis; 2017; 27:106-16. }\end{array}$} & \multirow{5}{*}{$\begin{array}{l}\text { randomized } \\
\text { counter- } \\
\text { balanced } \\
\text { repeated } \\
\text { measures }\end{array}$} & \multirow{5}{*}{ acute } & \multirow{5}{*}{$\begin{array}{c}22 \text { volunteers from seven } \\
\text { local running clubs; } 8 \text { female, } \\
\text { age } 33 \pm 8.3\end{array}$} & \multirow{5}{*}{\begin{tabular}{|}
$6000 \mathrm{~m}$ run indoors and \\
outdoors. two runs involving \\
both steady-state $3000 \mathrm{~m}$ \\
and high-intensity effort \\
$3000 \mathrm{~m}$ (self-selected)
\end{tabular}} & \multirow{5}{*}{$\begin{array}{c}\text { The outdoor green } \\
\text { condition was conducted } \\
\text { in a large woodland area, } \\
\text { with walking/running } \\
\text { trails lined with trees and } \\
\text { bushes. Dog walkers and } \\
\text { other runners occupied } \\
\text { the woodlands during } \\
\text { data collection }\end{array}$} & \multirow{5}{*}{$\begin{array}{l}\text { large fitness suite using a } \\
\text { Life Fitness } 95 \mathrm{Ti} \text { treadmill } \\
\text { (Life Fitness, UK) set at an } \\
\text { elevation gradient of } 1 \% \text { in } \\
\text { order to accurately reflect } \\
\text { the energetic cost of } \\
\text { running outdoors on a flat } \\
\text { surface }\end{array}$} & $\begin{array}{l}\text { Performance } \\
\text { efficacy }\end{array}$ & $\begin{array}{c}\text { time to complete } \\
\text { (minutes, seconds) }\end{array}$ & no differences \\
\hline & & & & & & & Well-being & Feeling Scale & no differences \\
\hline & & & & & & & $\begin{array}{l}\text { Performance } \\
\text { efficiency }\end{array}$ & Felt Arousal Scale & no differences \\
\hline & & & & & & & $\begin{array}{l}\text { Performance } \\
\text { efficiency }\end{array}$ & $\mid \begin{array}{c}\text { Rating of Perceived } \\
\text { Exertion }\end{array}$ & no differences \\
\hline & & & & & & & Well-being & $\mid \begin{array}{c}\text { Subjective Vitality } \\
\text { Scale }\end{array}$ & no differences \\
\hline & & & & & & & $\begin{array}{l}\text { Performance } \\
\text { efficiency }\end{array}$ & Heart Rate $(\mathrm{bpm})$ & no differences \\
\hline & & & & & & & $\begin{array}{l}\text { Performance } \\
\text { efficiency }\end{array}$ & $\begin{array}{c}\text { Rating of Perceived } \\
\text { Exertion }\end{array}$ & no differences \\
\hline
\end{tabular}




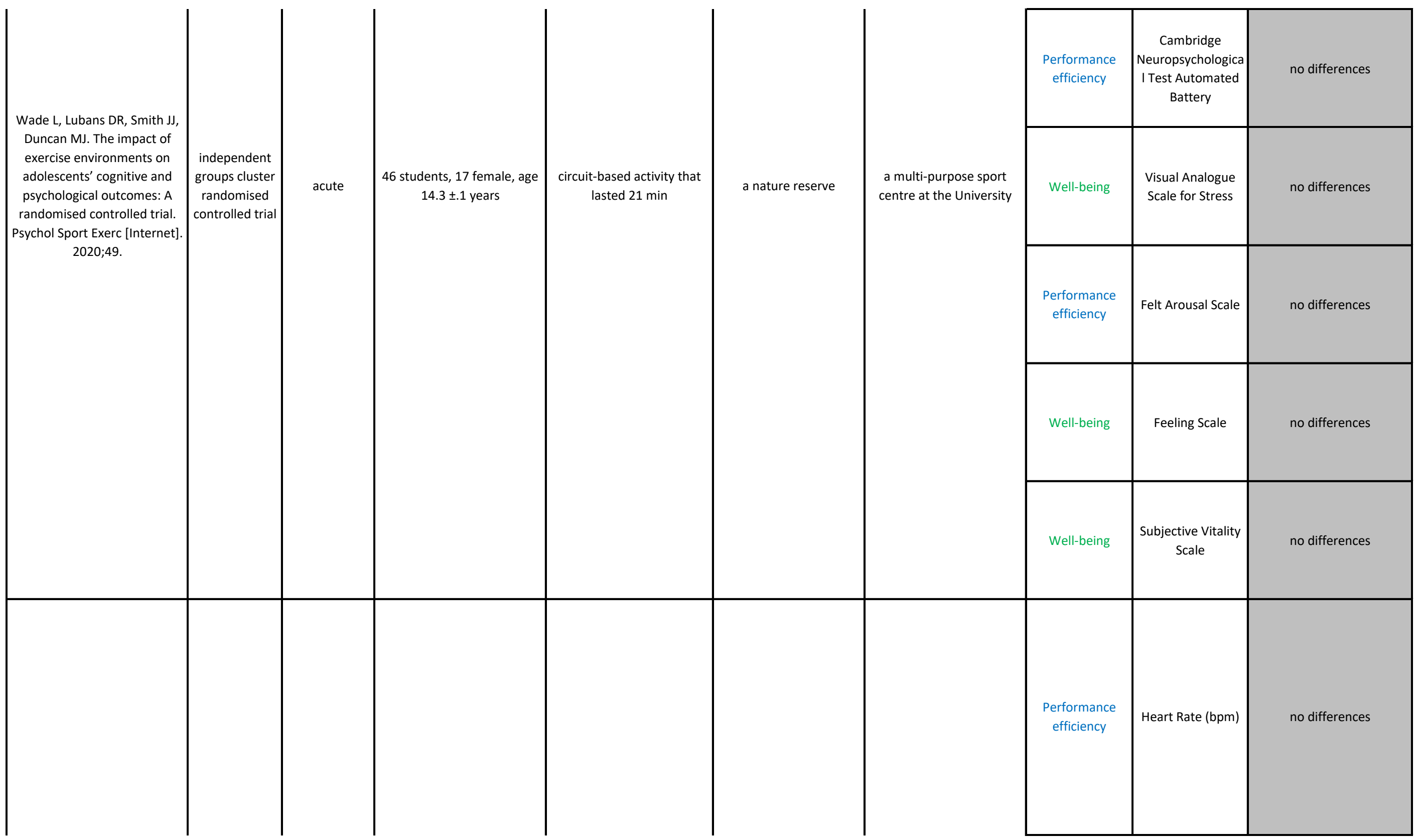


White MP, Pahl S, Ashbullby KJ, Burton F, Depledge MH.

The effects of exercising

on psycho-physiological outcomes in post-menopausa

women: A simulation study.

Int J Environ Res Public Health.

2015;12:11929-53.
Williams AL, Gill D. Perceived environmental restorativeness and affective responses to indoor vs. outdoor exercise. University of North Carolina at Greensboro; 2011. within-subjects acute (nature 37 post-menopausal women

repeated

measures simulation)
15 min cycle ergometer indoor in front of wall while wathing white screen or nature video. Intensity such that "you should feel comfortable holding a conversation during the ride, but at the end of the 15 min you may be slightly out of breath and slightly sweaty.

room with Green video featuring three $5 \mathrm{~min}$ scenes of fields with sheep, hedgerows and a small wood. The Blue video featured three 5 min clips from a headland

overlooking a beach and of views from beach height across rocks and the sea

6 young adult women randomized counterbalanced repeated measure acute recruited from the University of North Carolina a Greensboro, age $20.5 \pm 2.1$
Irwin Belk recreation track, a 636-meter paved 30 minutes jogging/walking maximum heart rate at $60-70 \%$ of age-predicted

\begin{tabular}{|c|c|c|c|}
\hline \multirow{3}{*}{ white screen in a room } & Well-being & Feeling Scale & $\begin{array}{l}\text { greater positive affect for } \\
\text { nature-based exercisers }\end{array}$ \\
\hline & $\begin{array}{l}\text { Performance } \\
\text { efficiency }\end{array}$ & Felt Arousal Scale & no differences \\
\hline & $\begin{array}{l}\text { Performance } \\
\text { efficiency }\end{array}$ & $\begin{array}{c}\text { Rating of Perceived } \\
\text { Exertion }\end{array}$ & no differences \\
\hline \multirow{4}{*}{$\begin{array}{l}\text { an indoor track was used } \\
\text { for the indoor condition. } \\
\text { This } 188 \text {-meter track in the } \\
\text { student recreation center is } \\
\text { suspended above three } \\
\text { recreational basketball } \\
\text { courts. }\end{array}$} & Well-being & Feeling Scale & no differences \\
\hline & $\begin{array}{l}\text { Performance } \\
\text { efficiency }\end{array}$ & Felt Arousal Scale & no differences \\
\hline & $\begin{array}{c}\text { Performance } \\
\text { efficiency } \\
\text { Well-being }\end{array}$ & $\begin{array}{c}\text { Activation- } \\
\text { Deactivation } \\
\text { Adjective Checklist }\end{array}$ & no differences \\
\hline & $\begin{array}{l}\text { Performance } \\
\text { efficiency }\end{array}$ & $\begin{array}{c}\text { Rating of Perceived } \\
\text { Exertion }\end{array}$ & $\begin{array}{l}\text { lower perceived exertion in } \\
\text { indoor exercisers }\end{array}$ \\
\hline
\end{tabular}




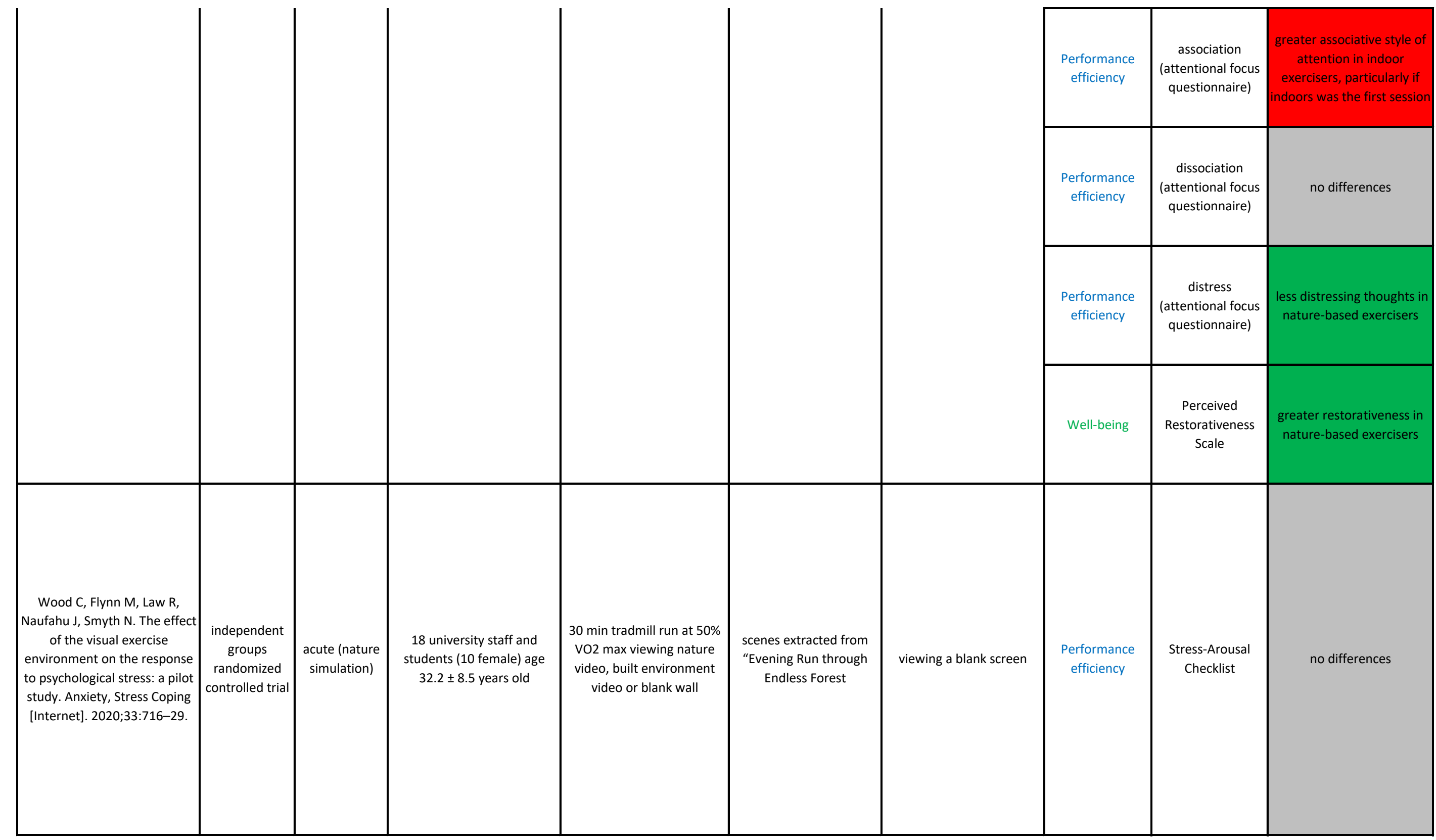




\begin{tabular}{|c|c|c|c|c|c|c|c|c|c|}
\hline $\begin{array}{l}\text { Wooller JJ, Rogerson M, } \\
\text { Barton J, Micklewright D, } \\
\text { Gladwell V. Can simulated }\end{array}$ & $\begin{array}{c}\text { randomized } \\
\text { independent }\end{array}$ & acute (nature & 20 healthy paticipants, age & 5 minute cycling at $40 \%$ & simulated nature sounds & without nature simulation & $\begin{array}{l}\text { Performance } \\
\text { efficiency } \\
\text { Well-being }\end{array}$ & $\begin{array}{c}\text { total mood } \\
\text { disturbance } \\
\text { (Profile of Mood } \\
\text { States) }\end{array}$ & $\begin{array}{c}\text { lower total mood } \\
\text { disturbance in nature-based } \\
\text { exercisers }\end{array}$ \\
\hline $\begin{array}{l}\text { recovery from acute mental } \\
\text { stress? Front Psychol } \\
\text { [Internet]. 2018;9. }\end{array}$ & $\begin{array}{c}\text { groups } \\
\text { controlled trial }\end{array}$ & simulation) & $\mathrm{n} / \mathrm{a}$ & output (EPPO) & and video combined & & Well-being & $\begin{array}{l}\text { Perceived Stress } \\
\text { Scale }\end{array}$ & $\begin{array}{l}\text { lower stress in nature- } \\
\text { based exercisers }\end{array}$ \\
\hline $\begin{array}{l}\text { Yeh H-P, Stone JA, Churchill } \\
\text { SM, Brymer E, Davids K. } \\
\text { Physical and Emotional } \\
\text { Benefits of Different Exercise }\end{array}$ & randomized & acute (nature & 30 regular exercisers, 12 & $\begin{array}{l}20 \text { minute run viewing video } \\
\text { of nature while on the } \\
\text { treadmill, or Self-Selected }\end{array}$ & $\begin{array}{l}\text { Nature video was } \\
\text { recorded at the Sheffield } \\
\text { Botanical Gardens. The } \\
\text { video represented a first }\end{array}$ & $\begin{array}{l}\text { Entertainment Condition } \\
\text { involving participants } \\
\text { running with their } \\
\text { preferred, self-selected } \\
\text { entertainment, such as }\end{array}$ & $\begin{array}{l}\text { Performance } \\
\text { efficiency }\end{array}$ & $\begin{array}{c}\text { Energy } \\
\text { Expenditure (kcal) }\end{array}$ & no differences \\
\hline $\begin{array}{l}\text { Environments Designed tor } \\
\text { Treadmill Running. Int J } \\
\text { Environ Res Public Health. } \\
\text { 2017;14. }\end{array}$ & $\begin{array}{l}\text { repeated } \\
\text { measures }\end{array}$ & simulation) & female, age $27.5 \pm 9$ & $\begin{array}{l}\text { involving participants } \\
\text { running with their preferred } \\
\text { (self-selected intensity) }\end{array}$ & $\begin{array}{c}\text { person perspective of } \\
\text { running through the } \\
\text { gardens, filmed at } 5.2 \\
\mathrm{mph}\end{array}$ & $\begin{array}{l}\text { watching a TV programme } \\
\text { on a wall-mounted } \\
\text { monitor, as they would in a } \\
\text { gymnasium }\end{array}$ & Well-being & $\begin{array}{l}\text { Positive and } \\
\text { Negative Affect } \\
\text { Scale }\end{array}$ & no differences \\
\hline
\end{tabular}

